\title{
QCD challenges from pp to A-A collisions
}

\author{
J. Adolfsson ${ }^{1}$, A. Andronic ${ }^{2}$, C. Bierlich ${ }^{3,18}$, P. Bozek ${ }^{4}$, S. Chakraborty ${ }^{3}$, P. Christiansen ${ }^{1, a}$, D. D. Chinellato ${ }^{5}$,
} R. J. Fries ${ }^{6}$, G. Gustafson ${ }^{3}$, H. van Hees ${ }^{7}$, P. M. Jacobs ${ }^{8}$, D. J. Kim ${ }^{9}$, L. Lönnblad ${ }^{3}$, M. Mace ${ }^{9,10}$, O. Matonoha ${ }^{1}$, A. Mazeliauskas ${ }^{11,12}$, A. Morsch ${ }^{12}$, A. Nassirpour ${ }^{1}$, A. Ohlson ${ }^{1}$, A. Ortiz ${ }^{13}$, A. Oskarsson ${ }^{1}$, I. Otterlund ${ }^{1}$, G. Paić ${ }^{13}$,

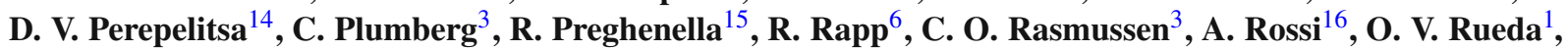
A. V. D. Silva ${ }^{5}$, D. Silvermyr ${ }^{1}$, A. Timmins ${ }^{17}$, T. Sjöstrand ${ }^{3}$, R. Törnkvist ${ }^{3}$, M. Utheim ${ }^{3}$, V. Vislavicius ${ }^{18}$, U. A. Wiedemann ${ }^{12}$, K. Zapp ${ }^{3}$, W. Zhao ${ }^{19}$

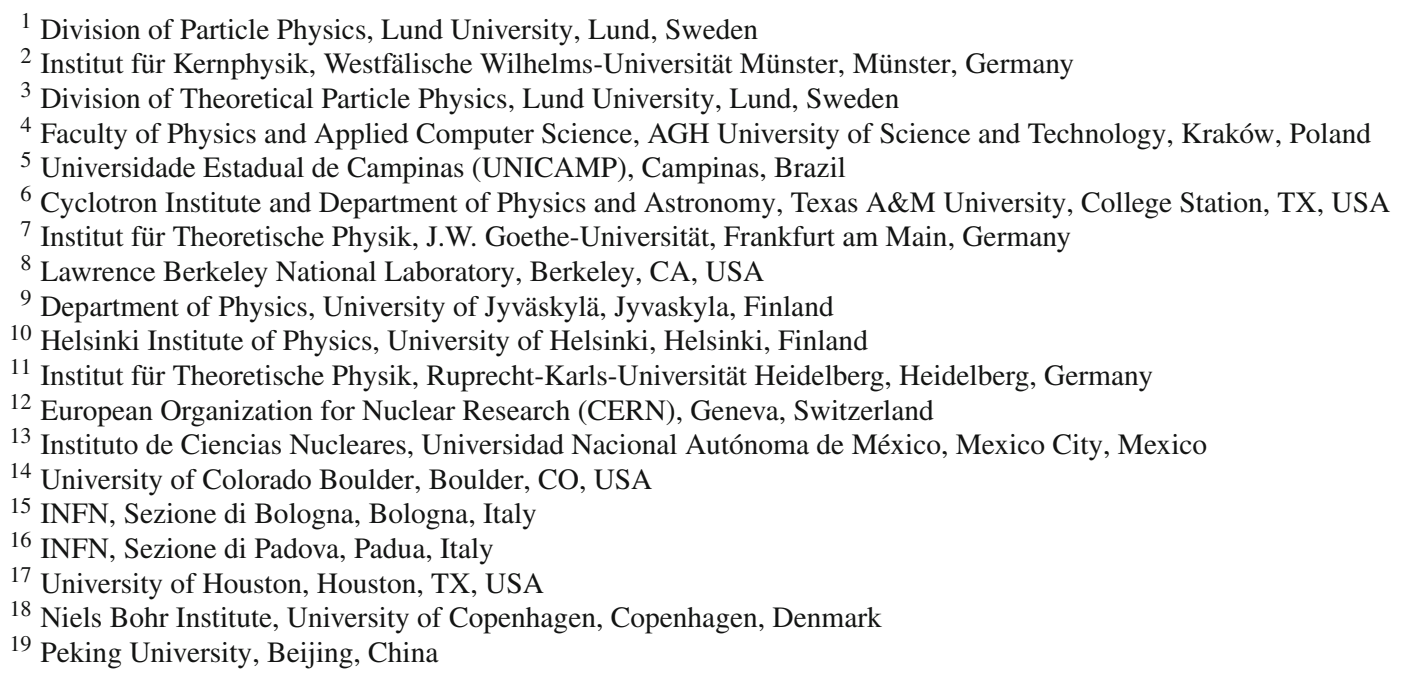

Received: 24 March 2020 / Accepted: 27 September 2020

(C) The Author(s) 2020

Communicated by Nicolas Alamanos

\begin{abstract}
This paper is a write-up of the ideas that were presented, developed and discussed at the third International Workshop on QCD Challenges from pp to A-A, which took place in August 2019 in Lund, Sweden (Workshop link: https://indico.lucas.lu.se/event/1214/). The goal of the workshop was to focus on some of the open questions in the field and try to come up with concrete suggestions for how to make progress on both the experimental and theoretical sides. The paper gives a brief introduction to each topic and then summarizes the primary results.
\end{abstract}

\section{Introduction}

The Quark-Gluon Plasma (QGP) is a phase of QCD matter at high temperatures in which quarks and gluons are deconfined. As the temperature of the phase transition is in

a e-mail: peter.christiansen@hep.lu.se (corresponding author) a regime where $\mathrm{QCD}$ is non-perturbative, providing quantitative theoretical calculations of QGP properties in hadronic collisions is challenging. For this reason, the characterization of these properties has come mainly from experimental observations in ultra-relativistic collisions of gold nuclei at RHIC and lead nuclei at the LHC. Thus, while the QGP phase has well defined properties in, for example, lattice QCD, the QGP paradigm used in the research related to the experiments at RHIC and LHC is based mainly on the need to provide a unifying explanation of many physical phenomena, the most prominent of which include jet quenching, multiparticle long-range flow correlations, strangeness "enhancement", charmonium/bottomonium suppression, open heavyflavor diffusion and electromagnetic radiation. In recent years, QGP-like observations have also appeared to manifest themselves in small systems [1]. In particular, significant effects are observed with increasing multiplicity in these collision systems, such as stronger long range $\Delta \eta$ correlations, magnitude and sign of multi-particle flow cumulants which 
are consistent with hydrodynamic flow, an enhancement in strangeness production relative to non-strange hadrons, elliptic flow of heavy flavor hadrons, and increasing baryon-tomeson ratios at intermediate $p_{\mathrm{T}}$. It remains an open and important question whether these observations require a QGP explanation, or can be described by other physical mechanisms. At the same time, some of the typical dense QCD medium effects, e.g., jet-quenching or heavy flavor $R_{\mathrm{AA}}$ modification, have not yet been observed in small collision systems. Finally, the observation of QGP-like effects in small systems also provides new input and directions for the interpretation of the phenomena in large systems.

The goal of the workshop was to discuss five concrete topics:

- Can we get the initial state to reveal itself?

- In what ways are QGP-like effects in small systems related to each other?

- Is there jet quenching in small systems, and can we measure and calculate it?

- How does the hadronization process depend on the properties of the hadronizing system?

- Can heavy quarks unravel common mechanisms in small and large systems?

Each participant at the workshop was assigned to a unique topic and prepared a poster related to this topic. First, the posters were all discussed in a plenary session. The topical posters were then discussed within the smaller topical groups with the goal to identify open questions and concrete ideas for making progress on each topic. The questions and ideas were then discussed both within the topical groups, as well as in meetings between each of the topical groups. Finally, the main ideas and discussions were summarized in a plenary session. This write-up is based on these final presentations and has been organized so that each section represents one topic. The goal has been to present each topic in a way that it can be read on its own, meaning that sometimes information is repeated.

\section{Can we get the initial state to reveal itself?}

In this section, we consider the ways in which the initial state and subsequent stages of a hadronic collision may imprint themselves onto final-state observables, particularly in ways which are relevant to distinguishing competing models of the different stages of evolution in these collisions.

Small collision systems ( $p p, \mathrm{p}-\mathrm{A}$ ) have historically been used to study initial and final state effects in "cold" nuclear matter, in order to establish a baseline for the interpretation of heavy-ion (A-A) results. Comparisons with this baseline have lead to the establishment of "hot" medium effects in
A-A collisions, such as jet quenching [2], quarkonium suppression and regeneration [3], strangeness enhancement [4], and collective flow [5], all of which together provide strong evidence for the production of a color deconfined medium, the QGP.

However, in recent years collective, fluid-like features strikingly similar to those observed in heavy-ion collisions, such as long-range correlations [6-8] and the increase of strange particle yields with charge particle multiplicity [9], have been also observed in small collisions systems. The question then arises whether QGP is also created in these small systems or, conversely, whether some alternative mechanism could explain the observations in all systems simultaneously. For example, theoretical modeling of the initial state within the Color-Glass Condensate (CGC)/saturation physics framework [10] and the subsequent space-time evolution (using kinetic theory [11] or event generators [12]) has suggested alternative potential descriptions of collective phenomena in these systems, which do not require the formation of a strongly coupled, deconfined plasma which evolves hydrodynamically.

For this reason, a wide variety of different approaches have become available for modeling the stages of relativistic nuclear collisions, with each approach offering a unique way of understanding the microscopic properties of these systems. Developing ways to discriminate between these approaches is clearly of paramount importance to the task of disentangling the origins of collectivity in nuclear collisions. In the present write-up, we consider three distinct approaches which are frequently discussed in connection with and employed in the modeling of small-system collectivity, and we propose several promising avenues for discriminating between them on the basis of theory, phenomenology, and experiment.

\subsection{Microscopic and macroscopic approaches}

A usual approach for constructing predictions of a given final state, is to combine an initial state and a final state calculation. The most common final state calculation is hydrodynamics [13], which offers an effective description formulating the system's dynamical and space-time evolution in terms of relativistic fluid dynamics by coarse-graining over microscopic degrees of freedom. Examples include iEBE-VISHNU [14] and MUSIC [15]. A recent alternative description is offered by string models including interactions between strings [16], to allow for a similar spatio-temporal evolution in a microscopic way. The initial state can similarly be constructed using different model assumptions. Simple assumptions in, e.g., Pythia/Angantyr [17,18] or HIJING [19] use a smooth distribution of multi-parton interactions (MPIs) in each nucleon. More elaborate frameworks, such as the Mueller dipole formalism [20,21], calculate a spatial 
Table 1 Classifying initial- and final-state models according to whether they are based on microscopic or macroscopic descriptions of system properties

\begin{tabular}{lll}
\hline & Microscopic & Macroscopic \\
\hline Initial state & Mueller dipoles, CGC & $\begin{array}{l}\text { CGC+hydro, } \\
\text { QCD kin. theory }\end{array}$ \\
Final state & String interactions & Hydrodynamics \\
\hline
\end{tabular}

distribution of gluons in the individual nucleons, and thus includes more fluctuations. A related framework is that of the CGC [10]. As a framework derived formally as a high-energy effective theory of QCD, it yields classical field theory equations, renormalization group equations, etc. It is extended by modeling to a finite nucleus in the IPGlasma model [22].

These approaches may be classified according to whether they are based on microscopic or macroscopic descriptions of the system's properties, as well as whether these properties are taken to originate in the initial or final stages of the collision, as shown in Table 1.

\subsection{Discriminators}

Our goal here is to identify several opportunities for discriminating between these different frameworks on the basis of experimental, phenomenological, and theoretical evidence, or some combination thereof. We center the discussion around observables which we consider to be especially promising in this regard. In particular, we consider the value in more careful analyses of multiplicity distributions, flow in small systems, and intensity interferometry in small systems.

\subsubsection{Multiplicity distributions}

Fluctuations in the total event-by-event multiplicity are sensitive in a unique way to both the multiplicity distributions themselves as well as to the jet pedestal (also called the Underlying Event). Moments of these distributions may therefore provide critical insights into the microscopic degrees of freedom at play in large and small collisions, particularly in their ability to constrain the still poorly understood initial state in pp collisions and in ultra-central nuclear collisions. It has been noted by ALICE [23], that the charged multiplicity per participant nucleon, defined by $2\left\langle\mathrm{~d} N_{\mathrm{ch}}\right\rangle / d \eta /\left\langle N_{\text {part }}\right\rangle$, shows an 'uptick' for limiting (high) values of $N_{\text {part }}$, breaking participant scaling. The same effect has been measured by CMS [24] and PHOBOS [25], but without giving it too much attention. The effect can be reproduced by some, but not all, models. While studies of multiplicity fluctuations are an active research area, there has, to our best knowledge, not been given too much attention to multiplicity fluctuations specifically for ultra-central heavy

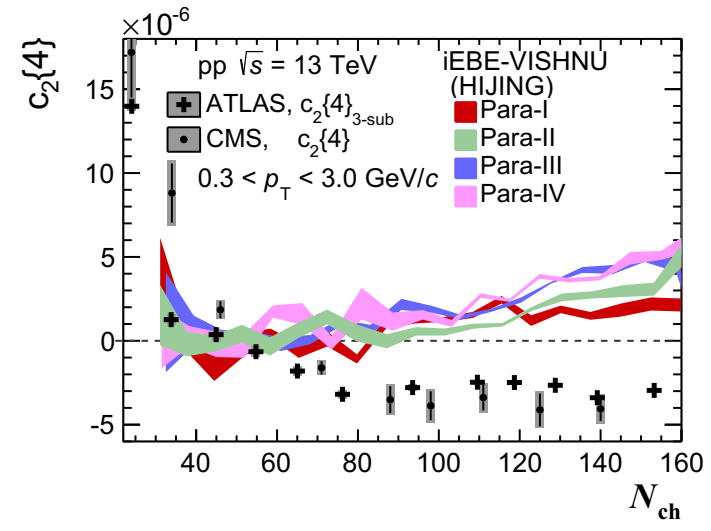

Fig. 1 The four-particle cumulant $c_{2}\{4\}$ as a function of $N_{\mathrm{ch}}$ for $\mathrm{pp}$ collisions at $13 \mathrm{TeV}$, as measured by the ATLAS [26] and CMS [27] collaborations and computed using HIJING + iEBE-VISHNU for several different parameterizations of the initial conditions. The theoretical calculations clearly generate $c_{2}\{4\}$ with the wrong sign for $N_{\mathrm{ch}} \gtrsim 60$. Taken from Ref. [28]

ion collisions. A more differential study, as well as a study of the scaled variance $\left\langle N_{\mathrm{ch}}^{2}\right\rangle /\left\langle N_{\mathrm{ch}}\right\rangle^{2}$, in the centrality bins where participant scaling is broken, are excellent candidates for observables for quantifying the effects of initial state fluctuations. To allow such studies, experimentalists are highly encouraged to publish multiplicity distributions, $p_{\mathrm{T}}$ distributions, and their joint distributions, in the highest-multiplicity collisions. This will place strong constraints on viable theoretical models of the initial state. To further narrow down the sources of fluctuations needed to understand breaking of participant scaling, theorists are similarly encouraged to attempt to reproduce these same experimental fluctuation studies using as few independent sources of fluctuations as possible. On both sides, these analyses should be repeated for longitudinal correlations as a function of multiplicity and $p_{\mathrm{T}}$ (cf. studies of flow-plane decorrelation), which are sensitive to both the breaking of boost invariance and the subsequent space-time evolution of the system.

\subsubsection{Flow in $p p$}

Current hydrodynamic approaches have difficulty in reproducing the experimentally observed negative sign of the fourparticle flow cumulant $c_{2}\{4\}$ in pp collisions [28], despite the use of a fluctuating initial state characterized by a negative eccentricity $\epsilon_{2}\{4\}$ [29]. An example of this situation is illustrated in Fig. 1. This suggests the possibility that final-state models (e.g., hydrodynamics), which typically generate an approximate $v_{2}\{4\} \propto \epsilon_{2}\{4\}$ scaling, either fail to describe pp collisions or fail to exhibit the usual linear-response behavior [30]. Another possibility is that the initial state geometry of pp collisions is so poorly modeled by existing approaches, that adding a correct response mechanism will fail to repro- 
duce data. Finally, it is also a possibility that contributions to multi-particle correlations from non-flow sources, in spite of experimental attempts to suppress such contributions, contaminates the signal to a degree where a model that does not correctly add such contributions, will inevitably fail. Several further questions can as such be addressed by the theory community. Most importantly it should be assessed whether any model for final state response (hydrodynamics, string shoving, etc.) can map a negative (toy geometry) $\epsilon_{2}\{4\}$ to a negative $c_{2}\{4\}$ using otherwise realistic model values for a pp collision. Furthermore, it should be investigated if and how CGC or interference based calculations [31] can generate a negative $c_{2}\{4\}$ in pp collisions. In the case of CGC, one should also understand the interplay between initial- and final-state effects by including the response from the final state to the $\epsilon_{2}\{4\}$ generated by a CGC treatment. On the experimental side, further investigations of quantities like flow fluctuations, also in p-A [32], symmetric cumulants in pp [33], and flow-harmonic correlations in all systems [3436], aim to further constrain models. To facilitate easier direct comparison between the often quite involved experimental observables and theory predictions, the use of collaborative comparison tools such as Rivet [37] is highly encouraged.

\subsubsection{Intensity interferometry}

The Hanbury Brown-Twiss (HBT) radii, $R_{i}^{2}(i=$ out, side, long), derived from two-hadron intensity interferometry [38], probe both the dynamical (momentum-space) and the spacetime (coordinate-space) structure of particle production in nuclear collisions. For this reason, they may also exhibit non-trivial effects of collectivity. Several well-documented features, present in both data and theoretical analyses, are of note, including a scaling with the transverse pair momentum $\left(k_{\mathrm{T}}\right)$, the breaking of scaling with the transverse pair mass $\left(m_{\mathrm{T}}\right)$, and the $\mathrm{d} N_{\mathrm{ch}} / \mathrm{d} \eta$-dependence of $R_{i}^{2}$.

Both $k_{\mathrm{T}}$-scaling and the breaking of $m_{\mathrm{T}}$-scaling affect the behavior of the $R_{i}^{2}$ at large $k_{\mathrm{T}}$. On the one hand, $k_{\mathrm{T}}$-scaling implies that the $R_{i}^{2}$ should decrease as $1 / k_{\mathrm{T}}$ in the presence of strong, transverse collective expansion [39-41]. On the other hand, in the case of $m_{\mathrm{T}}$-scaling, one expects the $R_{i}^{2}$, obtained in a suitable coordinate system using different particle species, to exhibit identical $m_{\mathrm{T}}$-dependence in the absence of collective flow, but to shift apart in the presence of fluid-like expansion [42].

In addition, the HBT radii are naively expected to scale linearly with $\left(\mathrm{d} N_{\mathrm{ch}} / \mathrm{d} \eta\right)^{1 / 3}$ at fixed pair momentum, and this behavior has been observed experimentally in virtually all collision systems from pp to A-A $[43,44]$. However, the slopes exhibit a strong hierarchy (out $<$ side, long) in $\mathrm{pp}$ which is observed to a limited extent in $\mathrm{p}-\mathrm{A}$ and not at all in $\mathrm{A}-\mathrm{A}$, where the slopes are comparable in magnitude for the three radii. This slope hierarchy, along with other aspects of the $\mathrm{d} N_{\mathrm{ch}} / \mathrm{d} \eta$-dependence of the HBT radii, may reflect fundamental differences of the evolution of pp geometry with multiplicity from that of $\mathrm{A}-\mathrm{A}$, and therefore places nontrivial constraints on models of small-system collectivity.

Furthermore, while both $k_{\mathrm{T}}$-scaling and the breaking of $m_{\mathrm{T}}$-scaling have been explored and are readily understood within the context of hydrodynamics, it is crucial for alternative approaches to reproduce these signals as well [45]. In hydrodynamic approaches, the $\mathrm{d} N_{\mathrm{ch}} / \mathrm{d} \eta$-dependence of the HBT radii reflect features of the initial state and subsequent space-time evolution [43]. However, some features, such as the strong hierarchy discussed above, remain not very well understood and constitute an important open challenge to all leading approaches to modeling collectivity in small systems.

\section{In what way are QGP-like effects in small systems related to each other?}

Here we discuss how observables associated with different phenomena are related, and propose future studies that would potentially resolve some of the ambiguities regarding their interpretation.

\subsection{Anisotropic flow and strangeness enhancement}

An enhancement of strange particle yields in heavy-ion collisions (relative to minimum-bias (MB) pp collisions) is typically associated with QGP formation. This is due to the fact that the temperatures required for deconfinement are higher than the mass of the strange quark, allowing equilibration to occur quickly. The damping of observed anisotropic flow coefficients $v_{\text {n }}$ with increasing $n$ in heavy-ion collisions is consistent with viscous damping in a system expanding hydrodynamically with a small value of $\eta / s$ (therefore a small mean free path), which is also expected for (strongly coupled) deconfined matter. Both of these effects are observed in high-multiplicity $\mathrm{pp}$ and $\mathrm{p}-\mathrm{Pb}$ collisions at the LHC $[4,7,46,47]$.

However, it is critical to address whether these phenomena can emerge from alternative (non-QGP) physical mechanisms, such as in the string picture where deconfinement is not explicitly assumed [48]. Strings in this context are phenomenological representations of the QCD field at large distances. High-multiplicity events often produce regions of high string density in the transverse plane. A mechanism has been proposed where overlapping strings can combine to form "ropes", and this leads to an increase in the effective string tension, which enhances the production of strange particles as a function of multiplicity [49]. Interactions between strings push them to lower-density regions a string "shoving" mechanism, which can convert the initial spatial anisotropies into final momentum anisotropies in 
the distribution of produced particles [16]. Therefore measurements of kinematic observables like $v_{\text {n }}$ might not be sufficient to identify the nature of the QCD state in such collisions. It was demonstrated in large A-A systems that higher-order correlations of flow coefficients [50] provide more information about the initial state and hydrodynamic evolution than measurements of $v_{\mathrm{n}}$ alone [34]; it may prove that these higher order correlations will also be necessary to discriminate between descriptions of flow-like signals in small systems, although measurements of symmetric cumulants in small systems still present open and unresolved challenges [51].

In order to discriminate between the QGP and string pictures, we propose a number of studies. The first consists of measurements of two-particle correlations between identified particles in small systems. In the PYTHIA model, when implementing ropes, the associated correlation functions are different compared to the case where ropes are not implemented, as shown in Fig. 10 [52]. The PYTHIA model (without ropes) tends to describe these measured correlation functions poorly, so if the introduction of ropes improves the agreement with data, it would add further validation to this implementation of a string description. Generally, such comparisons also address how "local" or "global" the production of conserved quantum numbers is, and how this could differ between the QGP, where conserved numbers can diffuse in the deconfined state, and the string picture, which contains no such dynamics (see also the discussion in Sect. 5.2). The second study we propose involves measurements of higher-harmonic anisotropic flow coefficients in small systems. These have not yet been measured at RHIC or the LHC beyond the 4th order $\left(v_{4}\right)$. Viscous hydrodynamics has specific predictions regarding how these coefficients should decrease for $\eta / s$ values associated with QGP formation, and comparing these predictions and those from string shoving to data could help to discriminate between the two approaches. We note here that string model predictions of higher harmonics and more differential flow observables are currently underway, and a quantitative prediction is necessary to determine if these observables can distinguish between the underlying physics of the dynamics in small systems. Finally, the same data could also be compared to $v_{\mathrm{n}}$ predictions that invoke a parton escape mechanism [53], which would challenge the hydrodynamic interpretation.

\subsection{Radial flow and particle production mechanisms at intermediate $p_{\mathrm{T}}$}

The higher mean $p_{\mathrm{T}}$ values in $\mathrm{pp}$ and $\mathrm{p}-\mathrm{Pb}$ collisions compared to $\mathrm{Pb}-\mathrm{Pb}$ collisions at the same multiplicities, and the smaller femtoscopic radii in $\mathrm{pp}$ and $\mathrm{p}-\mathrm{Pb}$ compared to $\mathrm{Pb}-\mathrm{Pb}$, indicate a faster collective expansion in the smaller systems. Blast-wave fits to the light-flavor hadron spectra enable a transverse-expansion velocity $\left(\beta_{\mathrm{T}}\right)$ to be extracted [54]. One might wonder whether the extracted information from the light-flavor blast-wave fits could be used to "predict" the multiplicity evolution of heavy-flavor hadron spectra (with charm or bottom quarks) in small systems. If so, this would further demonstrate that heavier quarks participate in the collective motion of the medium, which is something that is observed in heavy-ion collisions where QGP formation is expected. Another issue is the particle production mechanism at intermediate $p_{\mathrm{T}}$, roughly in the range of $1-10 \mathrm{GeV} / c$. In heavyion collisions, it is believed that quark coalescence (during the phase transition from a QGP to a hadron gas) may play an important role. It enhances baryon-to-meson ratios, and leads to a splitting between baryon and meson $v_{\mathrm{n}}$ in this $p_{\mathrm{T}}$ region. Predictions invoking quark coalescence describe the measured identified particle $v_{2}$ values from $\mathrm{p}-\mathrm{Pb}$ collisions rather well in this region [55]. The same predictions implement contributions from jet fragmentation, which become larger at higher $p_{\mathrm{T}}$, and cause a turnover in both $v_{\mathrm{n}}$ and the baryon-to-meson ratios as a function of $p_{\mathrm{T}}$. The onset of this turnover may be sensitive to possible jet-quenching effects in small systems, and other mechanisms such as initial state correlations which also predict a turnover. The study of these signals in the context of string-based models require further improvements, notably implementation of non-parallel string interactions.

\subsection{Hadron production from hard processes}

Jet quenching in heavy-ion collisions leads to a suppression of the production of high- $p_{\mathrm{T}}$ particles relative to expectations from a linear superposition of nucleon-nucleon collisions (MB pp collisions). Such a suppression is characterized by measurements of $R_{\mathrm{AA}}$. No such suppression has been observed yet, given current experimental uncertainties, either in $\mathrm{MB}$ or in high-multiplicity $\mathrm{p}-\mathrm{A}$ collisions relative to pp collisions. Naively, this is surprising, as the small mean-free-paths implied by the hydrodynamic description of anisotropic flow in high-multiplicity p-A collisions suggest that also high-energy partons interact significantly and lose energy, leading to jet-quenching effects. Clarifying this situation deserves novel and precise measurements to experimentally identify possible jet-quenching effects. A recent model that incorporates both heavy-flavor hydrodynamics and jet quenching attempts to simultaneously describe heavy-flavor $v_{2}$ and $R_{\mathrm{AA}}$ [56]. While this model has been used to calculate predictions for $\mathrm{O}-\mathrm{O}$ and $\mathrm{Ar}-\mathrm{Ar}$ collisions, no attempt has been made for $\mathrm{p}-\mathrm{Pb}$ collisions. Predictions for $\mathrm{p}-\mathrm{Pb}$ might shed more light on the level of suppression expected for heavy-flavor hadrons, and should also be extended to the light-flavor sector. 


\subsection{Selection biases in small systems}

Selecting events with large multiplicities (relative to the average multiplicity) in heavy-ion collisions leads to a selection of events with smaller than average impact parameters. In small systems, it is believed such selections have less of an influence on selecting geometry, and more of an influence on selecting rare hard scatterings producing very large number of particles. A study of near-side peak properties from two-particle correlations of charged hadrons could explore the role of hard processes with respect to these biases [57]. At higher particle $p_{\mathrm{T}}$, this peak is expected to be influenced by jet fragmentation. Experimental results in pp collisions show that both the amplitude and the width of the peak are fairly constant with respect to the multiplicity. A complementary study using PYTHIA found that the average $p_{\mathrm{T}}$ of the leading particle in a jet is also approximately constant with multiplicity [58]. Another source of information is provided by experimental studies of pp events which have a charged particle with $p_{\mathrm{T}}>7 \mathrm{GeV} / c$ [59]. Previous theoretical work indicates such events would have smaller impact parameters (compared to those without such a selection), and therefore smaller initial-state eccentricities [60]. Naively, this would lead to smaller anisotropic flow coefficients, assuming such flow is generated by a hydrodynamic response to the initial-state geometry. The fact that no such effect is observed experimentally calls for events with charged particles that have a higher $p_{\mathrm{T}}$ to be explored in more depth. If the results from additional studies remain the same, they could prove challenging to assumptions of smaller impact parameters or hydrodynamic response.

\section{Is there jet quenching in small systems, and can we measure and calculate it?}

Jet quenching and collective flow are different manifestations of the same underlying physics, the final-state reinteraction of the degrees of freedom liberated in a high-energy collision, though at widely differing momentum scales. Can we relate quantitatively the rescattering effects on a $\mathcal{O}(100 \mathrm{GeV})$ component tested in jet quenching to rescattering effects on a $\mathcal{O}(1 \mathrm{GeV})$ component revealed in collective flow? The connection is complex, for instance due to the running of the coupling and the opening of inelastic channels with increasing momentum transfer, but its elucidation would provide a deep understanding of the dynamics of hot QCD matter.

In addition to different characteristic momentum scales, jet quenching and collective flow also have different characteristic spatial scales, as discussed below. A valuable tool to explore the connection between jet quenching and collective flow is therefore to vary the size of the collision system, by varying the mass of the nuclear projectiles. A systematic pro- gram with this approach has been undertaken at both RHIC and the LHC, with particular emphasis on "small" systems in which one or both of the projectiles is a proton or light nucleus.

Current collider measurements with $\mathrm{pp}, \mathrm{p}-\mathrm{A}$, and light nucleus-A collision systems all exhibit clear phenomena suggestive of the presence of collective flow, while jetquenching effects in these systems are smaller than current measurement uncertainties. In this section we consider the ways in which QCD collective dynamics might have such a strong $p_{\mathrm{T}}$-scale-dependence that very large collective flow signals could occur in conjunction with jet-quenching signatures which evade current measurement limits.

It is the nature of hadronic collisions that an ensemble of events recorded with a $\mathrm{MB}$ trigger comprises a broad spectrum of physics processes, such as momentum transfer between incoming partons, final-state multiplicities, and the like. We label as "Event Activity" (EA) the experimentally accessible observables characterizing an event, such as forward multiplicity or transverse energy, and carry out jet quenching and collective flow measurements as a function of EA. The crucial question is then the relationship between measured EA and theoretically accessible quantities such as impact parameter or number of MPIs. Only by addressing this question for small systems can we utilize theoretical jetquenching calculations to assess the expected magnitude of quenching effects in small systems, and their connection to A-A measurements.

Moreover, we need to understand the correlations between high- $p_{\mathrm{T}}$ triggers and low- $p_{\mathrm{T}}$ multi-particle production in small systems, and the extent to which these are driven by final-state interactions. This study will profit from a model implementation in which high- $p_{\mathrm{T}}$ and low- $p_{\mathrm{T}}$ particle production arise from the same dynamics, so that degrees of freedom at some scale need not be labeled "fluid" or "jet", but are just what they are: degrees of freedom at that scale.

In the following sections we discuss theoretical considerations of "smallness" from the points of view of kinetic transport and its fluid dynamic limit; a specific measurement of correlations between high- $p_{T}$ trigger and soft multi-particle production in small systems; and application of the Angantyr model to this measurement. While we focus here on specific examples, we aim to put them in context with other existing or proposed approaches and to sketch an approach towards more general understanding of small systems.

\subsection{QCD transport theory}

In kinetic-transport theory, high- $p_{\mathrm{T}}$ and low- $p_{\mathrm{T}}$ particle production can arise seamlessly from the same dynamics. Also, kinetic transport interpolates seamlessly between free streaming in the smallest and close-to-ideal fluid dynamics in the largest collision systems. These two features make kinetic 
transport of particular interest for a combined understanding of collective flow and jet quenching in small systems.

A paradigm shift about the applicability of transport theory to flow phenomena occurred when it was realized that for the collision kernel of QCD effective kinetic theory with strong coupling constant of realistic size, hydrodynamization can occur as fast as in strongly coupled field theories [61]. Also, multi-stage dynamical descriptions of heavy-ion collisions that include transport theory, such as AMPT, have been shown to account for flow phenomena. It was possible to tune AMPT such that it describes important benchmarks such as the integrated particle yields, momentum spectra, and $v_{2}$ of low- $p_{\mathrm{T}}$ pions and kaons in central and midcentral Au-Au collisions at $\sqrt{s_{\mathrm{NN}}}=200 \mathrm{GeV}$ and $\mathrm{Pb}-\mathrm{Pb}$ collisions at $\sqrt{s_{\mathrm{NN}}}=2.76 \mathrm{TeV}$ with reasonable accuracy [62]. However, it was also observed in further studies that AMPT does not quantitatively reproduce the higher-order flow harmonics of identified hadrons $\left(v_{2}, v_{3}, v_{4}\right.$, and $\left.v_{5}\right)$ in $\mathrm{Pb}-\mathrm{Pb}$ collisions at $\sqrt{s_{\mathrm{NN}}}=2.76 \mathrm{TeV}$ [63]; this was attributed to an underestimation of radial flow by $25 \%$ in AMPT compared to LHC measurements. These developments make it at least conceivable that a unified dynamical understanding of medium-effects in low- $p_{\mathrm{T}}$ and high- $p_{\mathrm{T}}$ particle production can be based on a transport theory with a QCD-based collision kernel.

Within such a transport model approach, the question of how large jet-quenching effects are in small systems can be reformulated as a two-step procedure: (1) How significant must the final state interactions be in the soft sector to account for the observed collective flow in small systems? (2) How strong must the destructive interference or the scaledependence of individual interactions for high- $p_{T}$ processes be, such that jet quenching in small systems could so far evade detection?

The first of these questions can be addressed in simplified kinetic theories in which, e.g., the response of elliptic flow to a given eccentricity is studied as a function of the opacity parameter, $\hat{\gamma}$ (a combination of energy density and transverse extension of the system). Several studies have established by now that even one single final-state interaction is efficient in building up sizeable flow effects, see Fig. 2, and may be sufficient to account for the observed flow in the smallest collision systems [53,64-67]. The question of how jet quenching fares in the limit of one or a few scatterings has been long since identified [68], but full model studies are still missing. The work in the present workshop did not focus on this question. The present subsection on QCD transport theory is included here to highlight that the open question of unifying the description of collectivity and jet quenching across system size is currently addressed by several approaches, including ones that differ significantly from the one documented in the remainder of this section.

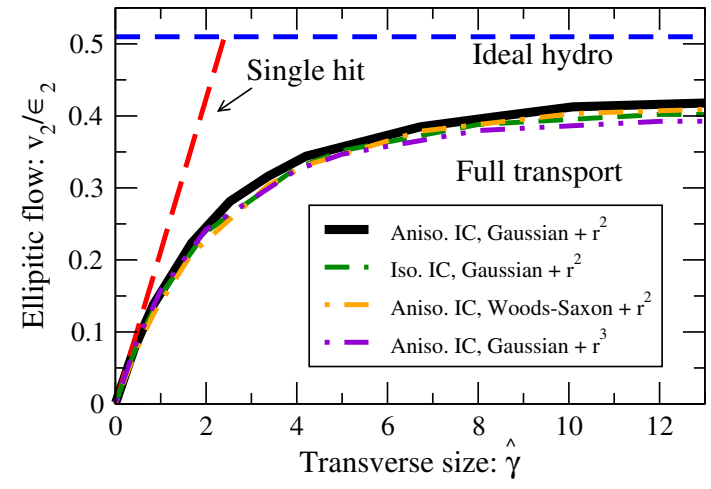

Fig. 2 Linear response $v_{2} / \epsilon_{2}$ of elliptic energy flow to eccentricity as a function of opacity $\hat{\gamma}$ in a conformal kinetic theory. One or very few scatterings can build up significant flow in small systems. Figure taken from Ref. [69]

\subsection{Experimental considerations}

\subsubsection{Jet-quenching observables}

Jet quenching arises from the disruption of the jet shower due to in-medium interactions. This generates several related effects for measurements of high- $p_{\mathrm{T}}$ hadrons and reconstructed jets:

- "Energy loss": medium-induced energy transport out of the acceptance of the observable. For high- $p_{\mathrm{T}}$ hadrons, "energy loss" corresponds to medium-induced radiation that depletes the energy of the (usually leading) shower branch which generates the hadron. For reconstructed jets it corresponds to medium-induced radiation transported outside the jet cone radius, $\mathrm{R}$;

- Medium-induced modification of jet substructure;

- Medium-induced acoplanarity, or deflection of the jet centroid.

All three types of effect must occur if any of them does, since they reflect different aspects of the same underlying physical processes.

Energy loss manifests itself phenomenologically as a yield suppression relative to measurements of the same observable in an unmodified reference system. Energy loss has been observed and measured extensively in A-A collisions using both inclusive and coincidence observables, and has been studied theoretically in depth.

Study of jet quenching via substructure modification is a recent development, based on tools adopted from the high-energy physics community [70]. Initial measurements indicate a modest medium-induced substructure modification $[71,72]$, though the experimental techniques are still under development and the theoretical interpretation of these modifications in terms of quenching is not yet firmly established. 
Medium-induced acoplanarity was the first proposed signature of jet quenching $[73,74]$ but has proven challenging to observe experimentally, beyond the broadening intrinsic to in-vacuo QCD. There are, however, new experimental and theoretical developments in this area [75-77].

The observation of jet quenching in small systems would be a major advance in our understanding of the formation and evolution of the QGP. As noted above, the most widely explored signature of jet quenching in $\mathrm{A}-\mathrm{A}$ collisions is energy loss, observed through measurements of yield suppression with both inclusive and coincidence observables. We now consider in turn each approach to searching for signatures of jet quenching in small systems.

\subsubsection{Inclusive jet measurements in small systems}

The most common observable sensitive to energy loss is $R_{\mathrm{AA}}$, the ratio of the yield measured in a complex collision system in which jet quenching occurs (e.g., central A-A collisions) to the scaled yield measured in a reference system, usually MB pp collisions. Scaling of the reference system yield is required to account for the trivial geometric effect that, in the absence of nuclear modifications, an A-A collision corresponds to many independent nucleon-nucleon collisions.

In general, each A-A collision can be characterized by EA (charged multiplicity, $E_{\mathrm{T}}$, etc.; measured at central or forward rapidities). In Fig. 3, the equivalent number of nucleonnucleon collisions ( $N_{\text {part }}$, for "number of participants") is calculated using Glauber modeling [79], which incorporates the nucleon distribution in the nucleus and a model of EA production, and generates a correlation between EA and $N_{\text {part }}$. The fact that this correlation becomes blurred for decreasing system size is a fundamental limitation for determining, on an event-by-event basis, the transverse geometrical extent of a small collision system.

In addition, in small systems there is a well-established correlation between the hard activity in the central region and the Underlying Event (UE), which biases EA as an estimator of collision geometry $[80,81]$. The various collaborations take different approaches to mitigate this effect for $\mathrm{p}-\mathrm{A}$ collisions: ALICE carried out model studies of the bias for different EA observables and concluded that the forward neutron energy, measured in a zero-degree calorimeter (ZDC), provides the least-biased EA metric [78]; PHENIX applies a correction for the bias based on Monte Carlo calculations [82,83]; and ATLAS does not correct for the bias [84].

Figure 4 shows EA-selected measurements of inclusive jet $R_{\mathrm{dAu}}$ by PHENIX [83] and $R_{\mathrm{pPb}}$ by ATLAS [84]. A strong dependence on EA is observed in both cases, with significant apparent yield enhancement for low EA and apparent yield suppression for high EA. In contrast, the MB distributions in these measurements (i.e., without EA selection) exhibit ratios $R_{\mathrm{dAu}}$ and $R_{\mathrm{pPb}}$ that are consistent with unity. Likewise,
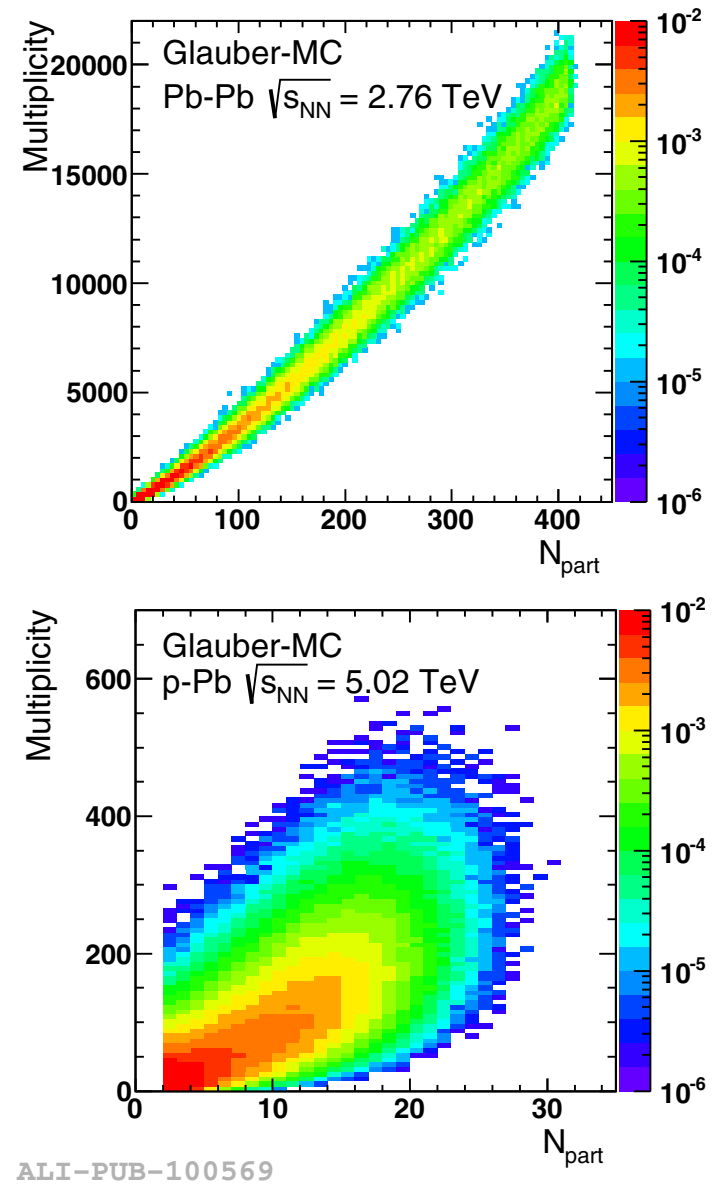

Fig. 3 Glauber-based calculations by ALICE of the correlation between forward charged-particle multiplicity (V0 detectors) and $N_{\text {part }}$ for $\mathrm{Pb}-\mathrm{Pb}$ collisions (top) and $\mathrm{p}-\mathrm{Pb}$ collisions (bottom) [78]

ALICE observes inclusive jet $R_{\mathrm{pPb}}$ consistent with unity for EA selections based on forward-neutron ZDC energy [85]. Finally, all EA-selected measurements of high- $p_{\mathrm{T}}$ inclusive hadron yields have $R_{\mathrm{pA}}$ consistent with unity [78,86-88].

It is evident that this set of EA-selected inclusive-yield measurements does not give a clear message about jet quenching in small systems. Several effects can contribute to apparent EA-dependent yield modifications, specifically initial-state effects and EA bias due to UE correlations with hard processes, in addition to jet quenching. Most importantly, EA-dependent inclusive-yield measurements have an irreducible dependence on Glauber modeling and its limitations in small systems. Further progress in the search for jet quenching in small systems requires alternative approaches.

\subsubsection{Coincidence jet measurements in small systems}

We now consider a coincidence observable that has potential to be sensitive to jet quenching but that avoids the need for Glauber modeling and the assumption that EA in 

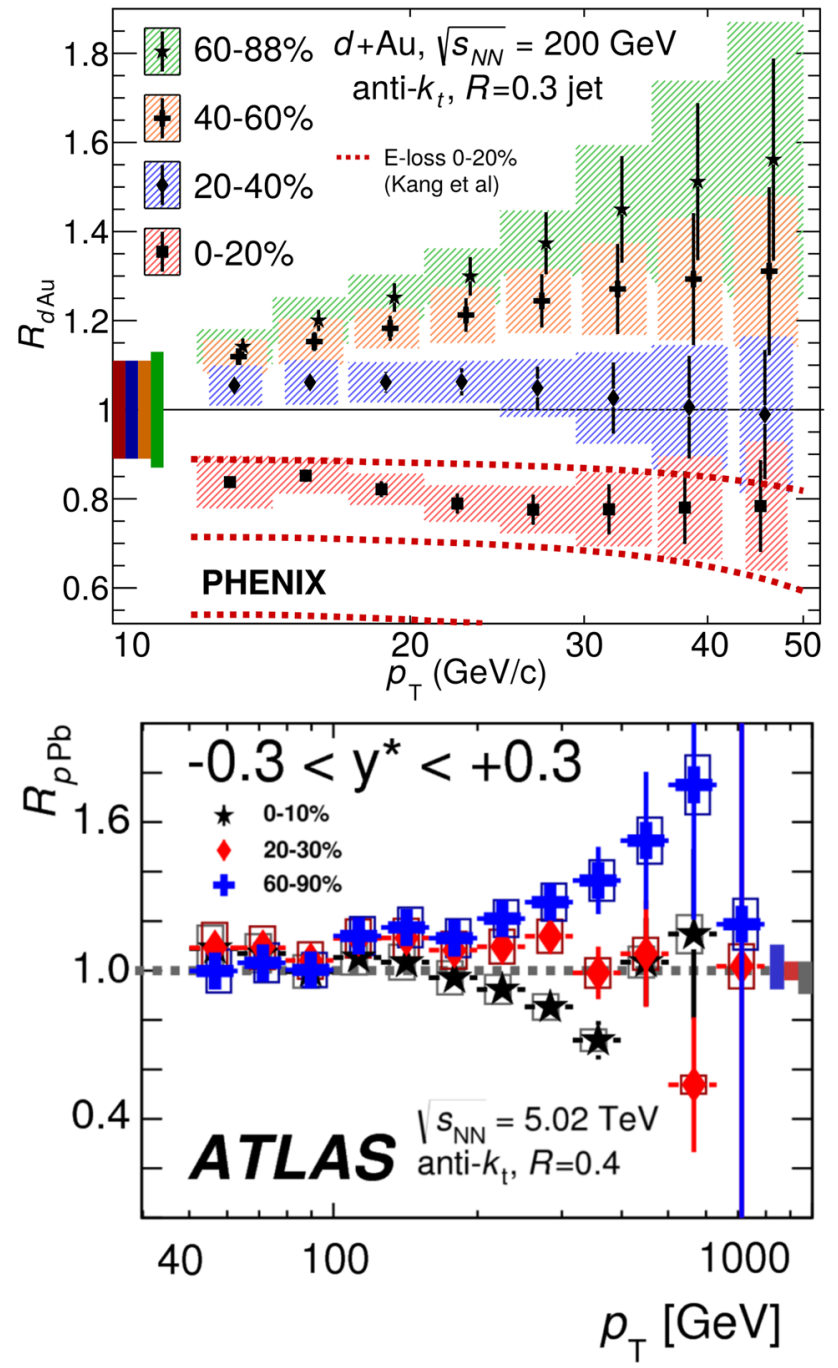

Fig. 4 Inclusive jet measurements in EA-selected $\mathrm{p} / \mathrm{d}+\mathrm{A}$ collisions. Top: $R_{\mathrm{dAu}}$ adapted from PHENIX [83]; bottom: $R_{\mathrm{pPb}}$ adapted from ATLAS [84]

small systems is correlated with collision geometry. The observable is the semi-inclusive distribution of jets recoiling from a high- $p_{\mathrm{T}}$ hadron, for EA-selected $\mathrm{p}-\mathrm{Pb}$ collisions at $\sqrt{s_{\mathrm{NN}}}=5.02 \mathrm{TeV}$ [89]. The EA is measured using forward charged-particle multiplicity in the $\mathrm{Pb}$-going direction (V0A detector, $2.8<\eta<5.1$ ).

Figure 5, top panel, shows the distribution of EA in decile bins of the V0A distribution. The blue points show EA for the MB population, which is trivially uniform in this representation: the decile bin boundaries as a function of V0A amplitude are chosen precisely so that this distribution is uniform. The red and black points show the EA (V0A) distribution for events with a high- $p_{\mathrm{T}}$ hadron in the central barrel, which biases towards large EA. This behavior is consistent with the well-known growth of the UE for events containing jets, in this case with the UE measured in the forward direction. We discuss Monte Carlo modeling of this bias in Sect. 4.3.2.
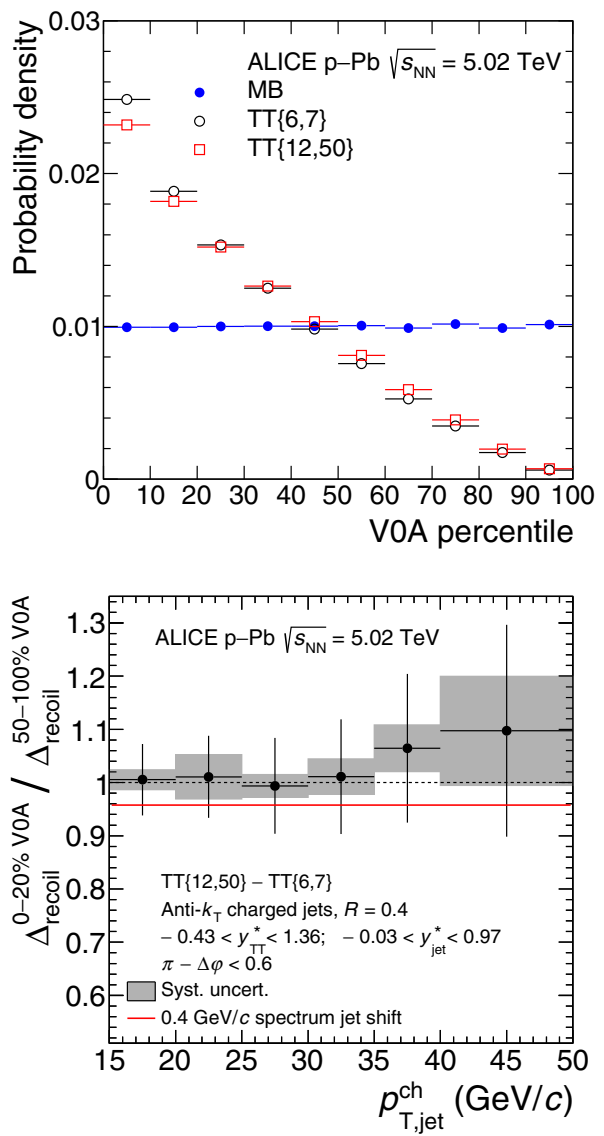

Fig. 5 Figures from Ref. [89]. Top: EA(V0A) distribution for the MB population, and event populations biased by the presence of a high- $p_{\mathrm{T}}$ hadron in the central barrel with $6<p_{\mathrm{T}}<7 \mathrm{GeV} / c(\mathrm{TT}\{6,7\})$ and $12<p_{\mathrm{T}}<50 \mathrm{GeV} / c$ (TT\{12,50\}). Decile bins chosen so the MB distribution is uniform. Large V0A amplitude (large EA) corresponds to small V0A percentile. Bottom: Ratio of semi-inclusive recoil jet yield for high and low EA. Red line is the ratio if the high-EA distribution would be shifted to lower $p_{\mathrm{T}, \text { jet }}^{\text {ch }}$ due to $p_{\mathrm{T}}$-independent energy loss of $400 \mathrm{MeV}$

Figure 5, bottom panel, shows the ratio of semi-inclusive recoil jet distributions for high-EA and low-EA event populations (see [89] for a discussion of the $\Delta_{\text {recoil }}$ observable). Energy loss due to jet quenching corresponds to a shift of the recoil jet distribution to lower $p_{\mathrm{T}, \text { jet }}^{\mathrm{ch}}$, with corresponding suppression of this ratio below unity if there are larger jetquenching effects in high-EA than low-EA collisions. The measured ratio is consistent with unity within uncertainties, indicating no significant jet-quenching effects in this measurement.

The measurement can, however, set a limit on jetquenching effects, in terms of the spectrum shift due to energy loss. Analysis, which takes into account the $p_{\mathrm{T},{ }^{\mathrm{cet}}{ }^{-}}{ }^{\mathrm{c}}$ dependence of the recoil distribution, gives a limit of $400 \mathrm{MeV}(90 \% \mathrm{CL})$ for the population-averaged mediuminduced charged-energy transport outside the jet cone radius 
of $0.4 \mathrm{rad}$, for high-EA relative to low-EA events (red line in figure) [89].

\subsection{Monte Carlo simulations}

In order to identify possible medium-induced jet modifications, it is imperative to carry out thorough event generator studies to make sure that every aspect of a measured observable is properly understood. Especially in small systems where event activity may be poorly correlated with the spatial extent of the medium in terms of impact parameter or number of participants (see, e.g., Fig. 3), it is important to understand possible biases that result from the selection of events. Ideally we would want event generators where one could switch on or off the medium effects, and although there are several programs available for A-A (see, e.g., Refs. [90,91]), proper modeling of jets in $\mathrm{p}-\mathrm{A}$ and $\mathrm{pp}$ collisions is still lacking.

For pp collisions we have general purpose event generators such as Herwig [92], PYTHIA [17,93], and Sherpa [94], which are able to give a good description of both hard and soft features of events. In PYTHIA ${ }^{1}$ this is achieved by a MPI scenario where the UE is treated in the same way as hard-jet production, i.e., starting from a soft or semi-hard perturbative scattering and adding initial- and final-state parton showers.

Within PYTHIA there are also (optional) models to include some collective effects, such as color reconnections [96], rope hadronization [49], and string shoving [16], introducing some cross-talk between different sub-scatterings in the MPI model used to describe the UE, but none of these specifically targets jet-quenching effects. Nevertheless, it has been shown [97] that default PYTHIA8 (which includes color reconnections, but no other collective effects) fairly well reproduces an observed effect that naively would have been interpreted as enhanced jet quenching in high-multiplicity pp jet events. Although the reason for this unexpected result is still not fully understood, it underlines the importance of event generators to help the interpretation of a given observable but also highlights the care with which these results have to be interpreted. On a similar note, recent studies of very high-multiplicity events in PYTHIA has found jet-quenching-like features that should be further investigated $[98,99]$.

\subsubsection{The Angantyr model}

For $\mathrm{p}-\mathrm{A}$ and $\mathrm{A}-\mathrm{A}$ collisions there is now an event generator model implemented in PYTHIA8 called Angantyr [18]. The main ingredients are an advanced Glauber model inspired by the so-called color fluctuation model [100-104] and an older generator called DIPSY [105-107], and the full power of

\footnotetext{
1 There are also similar models available for Herwig and Sherpa, see, e.g., Ref. [95], but we will here concentrate on PYTHIA.
}

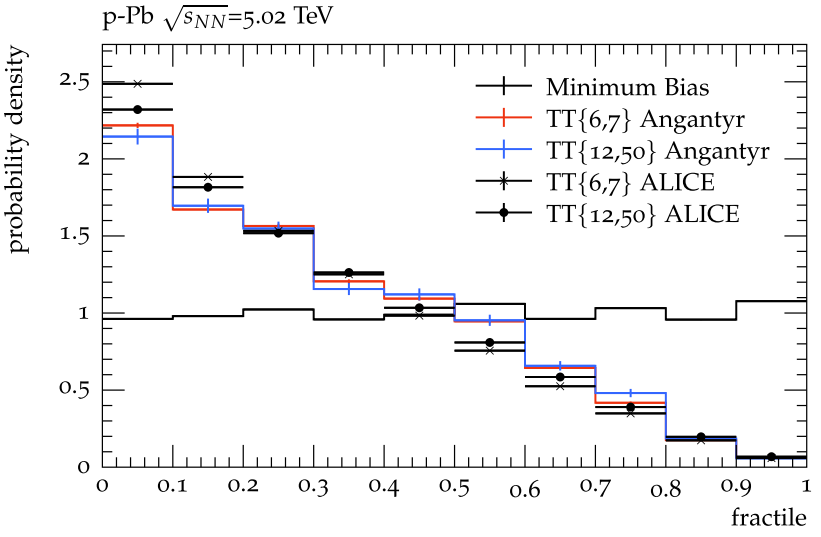

Fig. 6 Trigger bias in p-Pb from Angantyr vs. ALICE [89] (cf. Fig. 5, top panel)

the PYTHIA8 pp machinery to describe individual nucleonnucleon sub-collisions. The sub-collisions are currently simply stacked together into full $\mathrm{p}-\mathrm{A}$ or $\mathrm{A}-\mathrm{A}$ events using a model, inspired by the Fritiof program [108] and the MPI model in PYTHIA. In this way it extrapolates the dynamics of pp collisions to heavy-ion collisions, in a fairly simple way, without involving any collective effects between the sub-collisions. Nevertheless it is able to describe very well the measured rapidity distribution of charged particles in $\mathrm{p}-\mathrm{A}$ with very few tunable parameters [18], which then extrapolates to a very reasonable description of multiplicity distributions in A-A [18,23] without any further tuning.

Work has started to implement collective effects in Angantyr, but even without these the program can still be used to understand measurements. As an example it is shown in Ref. [18] that a non-zero $v_{2}$ is obtained in Angantyr, which then can be used to understand non-collective contributions to such an observable.

\subsubsection{Trigger bias calculations}

During this workshop a number of $\mathrm{p}-\mathrm{A}$ runs were made with Angantyr to try to shed light on the observed trigger-bias effect shown in Fig. 5. The results of the generator studies are presented in Fig. 6, and the bias found in data is very well reproduced by the generator results.

It is then possible to go into the underlying machinery of the generator and give more support to the statement that the bias for a $12-50 \mathrm{GeV} / c$ trigger hadron does not increase compared to a lower $p_{\mathrm{T}}$ trigger of 6-7 GeV/c. As an example we can inspect the model's impact-parameter distribution in a given centrality decile. This is shown in Fig. 7 where we first of all see the fairly poor correlation between the centrality measure used and the impact parameter. This comes as no surprise considering what we already saw in Fig. 3, and it becomes clear that the centrality measure is very sensitive to fluctuations in the final state. But we also see in the figure that 

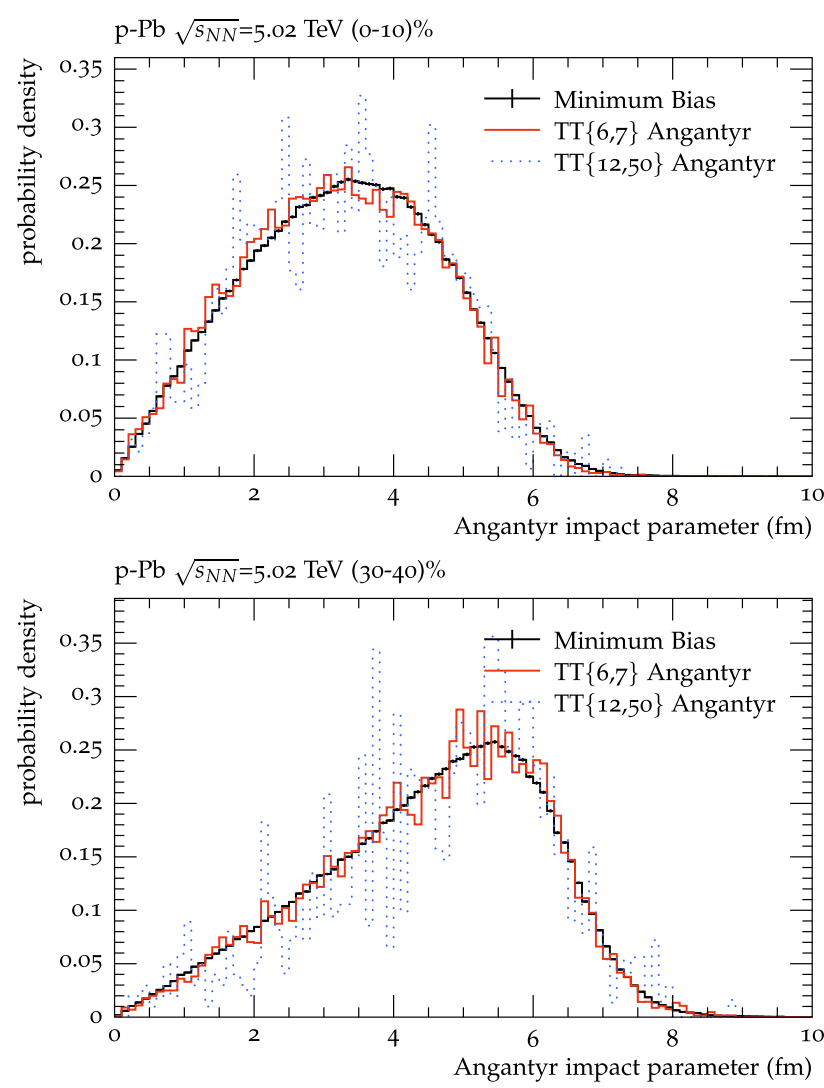

Fig. 7 Angantyr modeling of impact parameter distributions for two bins of event activity with and without a trigger hadron

the impact parameter distribution shows no significant sign of dependence on the trigger, indicating that the bias mainly comes from final-state fluctuations in the MPI generation rather than from the physical centrality of the collision.

\section{How does the hadronization process depend on the properties of the hadronizing system?}

For pp collisions, the general expectation has been that the hadronizing partons mainly reflect the initial partonic scatterings (including initial- and final-state radiation), with little or no additional final-state interactions before hadronization. Conversely, ultra-relativistic heavy-ion collisions are expected to produce a QGP in which quarks and gluons are deconfined, close to thermal equilibrium, and strongly interacting with each other up to the time of hadronization.

The observation of QGP-like effects in small systems presents two exciting opportunities: As hadronization models are challenged and new data become available, a great deal could be learned about the hadronization process itself, with profound connections to QCD confinement. On the other hand, as our understanding of hadronization grows we might be able to peel back this layer to learn about partons and their dynamics prior to hadronization.

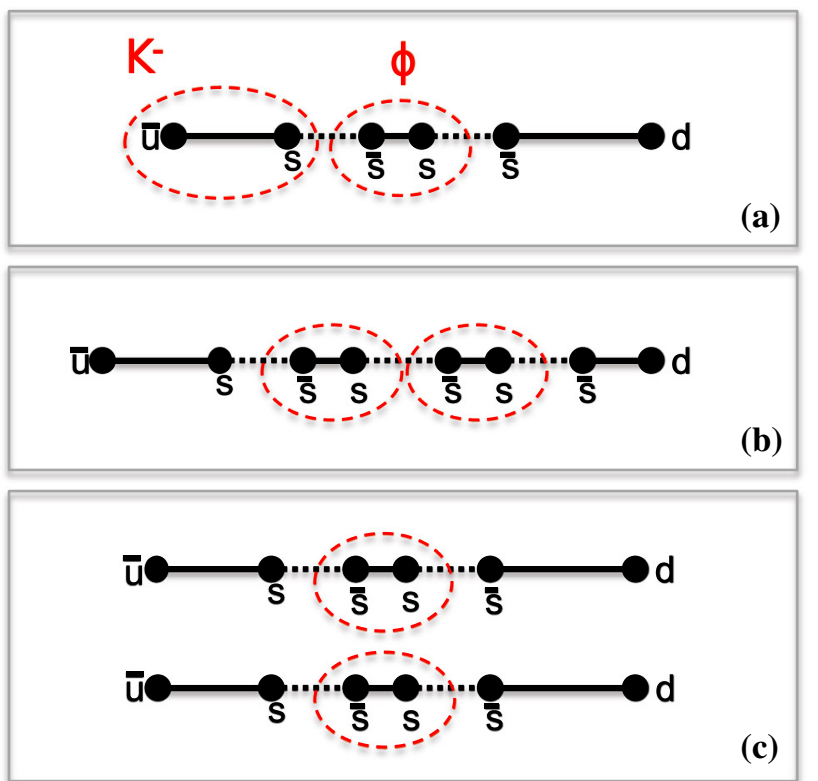

Fig. 8 Illustrations of $\phi$ and $\mathrm{K}^{-}$strange-meson creation in the Lund string picture. a String breakup into a $\phi$ and $\mathrm{K}^{-}$; b a single string breaking up into two $\phi$ mesons; $\mathbf{c}$ two strings breaking up into two $\phi$ mesons

A key idea that we focus on in the following is that in the string picture of hadronization [109], quantum numbers of produced hadrons are conserved locally. On the other hand, in a deconfined medium, or more generally a reservoir of partons, as implied by particle production in the grand-canonical limit, quantum numbers are not expected to be conserved locally. One would then rather describe hadrons by thermal models or by recombination of existing partons, at least some of which can come from the reservoir [110]. This idea will be discussed in the following two sections, which summarize the main outcomes of our discussions. A second focus point of the discussion is related to the space-time structure of jets and hadronization. This topic will be briefly elucidated in Sect. 5.3.

\subsection{Identified particle yield measurements}

The $\phi$ meson is an interesting case when comparing string models and thermal models. In the string models, the production of a $\phi$ meson requires two $s \bar{s}$-string breakings and is therefore doubly suppressed. On the other hand, in basic thermal models it is treated as a non-strange particle and the production is entirely driven by its mass.

A crucial point to realize is that once a $\phi$ meson has been produced by a long string, the production of a second $\phi$ meson next to it only requires one more $s \bar{s}$-string breaking, as illustrated in Fig. 8. This means that for a single long string, the string model would predict the following probabilities 


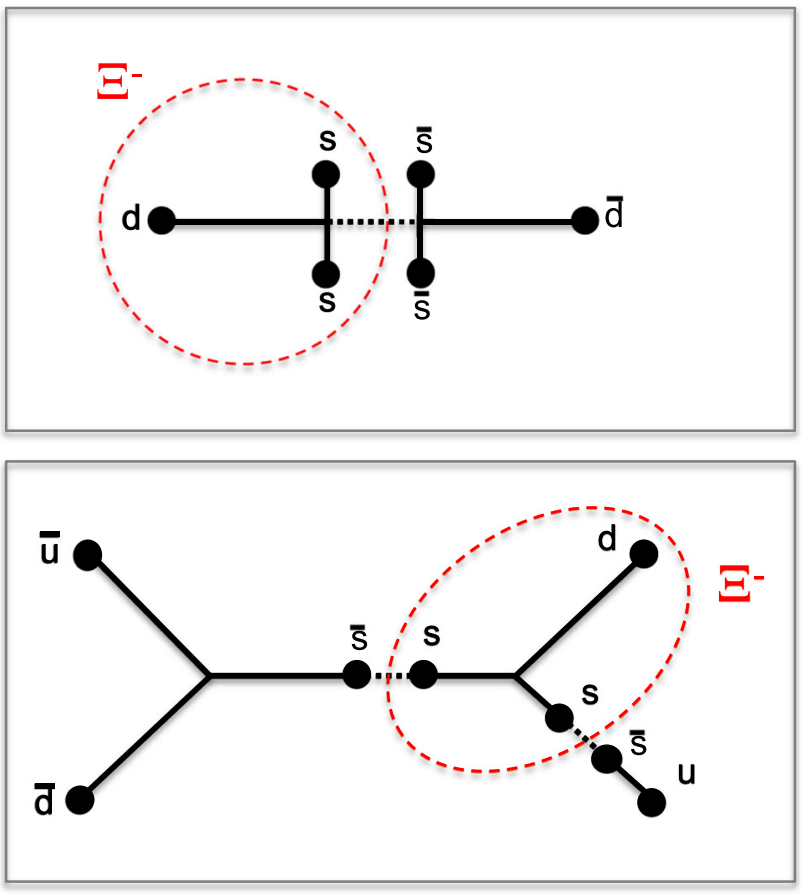

Fig. 9 Illustration of $\Xi$ production (top) in the standard Lund string framework, requiring diquark-anti-diquark pair production and (bottom) in a picture in which junctions are allowed to form

$$
\begin{aligned}
& P(\phi) \propto P_{s \bar{s}}^{2} \text { and } P(2 \phi) \propto P_{s \bar{s}}^{3} \\
& \quad \rightarrow P(2 \phi) \gg P(\phi)^{2} .
\end{aligned}
$$

It is worth pointing out here that this kind of advanced strangeness flow in strings was part of the validation of the string model using particle production in single-diffractive events [111].

The same kind of argument is true also for multi-strange baryons. In this case, as illustrated in Fig. 9, one must have a diquark-anti-diquark string breaking. In the case of a $s s \bar{s} \bar{s}$ breaking it is clear that one is very likely to produce $\Xi^{-}$and $\bar{\Xi}^{+}$together.

For MPIs, which can lead to event-to-event variations in the number of strings, the combinatorics is more complicated but the basic idea holds and detailed calculations can be made using event generators. One can use correlations, discussed in the next subsection, to separate correlated (same string) and uncorrelated (different string) production.

Finally, we want to add that the arguments presented here are also expected to apply for rope models [49]. For ropes, the probability to have $s \bar{s}$ and $s s \bar{s} \bar{s}$ breakings is enhanced but the local quantum-number conservation effects are the same as for strings.

\subsection{Per-trigger-yield measurements}

In this section we will refine some of the arguments of the previous subsection. The idea is that since strangeness is conserved by the strong interaction, one can to some degree recover the anti-strangeness associated with strangeness production using per-trigger-yield measurements. ${ }^{2}$ The advantage of using the per-trigger yield is that one can study the local strangeness production. This is not the case for correlation functions, which have been used to study similar properties [114] but where the magnitude of the correlation function is affected by the global strangeness production (it can for example be changed by increasing or decreasing the UE production). As we want to constrain the microscopic strangeness-production mechanism we think the per-triggeryield measurements will provide additional and more discriminative information. In the rest of this section we will mean per-trigger-yield measurements when we refer to correlations.

Correlations of the $\Xi^{-}(s s d)$ with other hadrons are one example, explored in string fragmentation in Fig. 9. Looking at the top of the figure, it is clear that one needs to have at least one $s$ quark in the diquark, so that the anti-baryon will have to be anti-strange. This means that if string or rope models are correct, then one should find that there are strong $\Xi^{-}-\bar{\Lambda}$ correlations and weak/vanishing $\Xi^{-}-\bar{p}$ correlations. One can subtract the combinatorial correlations from these, e.g., those introduced by minijets, by subtracting the samequantum-number correlations $\left(\Xi^{-}-\Lambda\right.$ and $\left.\Xi^{-}-p\right)$.

In PYTHIA, an important component in the phenomenological modeling is color reconnection, where several schemes have recently been explored [115]. One of the schemes that can give quite different correlations is the baryon junction scheme [113] illustrated in the bottom plot of Fig. 9. In the baryon junction scheme there is no longer a "requirement" that the associated anti-baryon of a $\Xi^{-}$is anti-strange. In that case, one will also produce substantially fewer pairs of $\Xi^{-}$and $\bar{\Xi}^{+}$. The important point to stress here is that there are clear strangeness-production fingerprints in microscopic models, which can be tested by measuring such correlations.

At the recent Quark Matter 2019 conference, ALICE has presented the first preliminary measurements of $\Xi-\pi$ and $\Xi-K$ correlations. As these results are preliminary only we show here the simulation results (cf. Fig. 10; upper and lower panel, respectively). The simulation results are shown for PYTHIA8 [17,93] and EPOS-LHC [112], which have very different production mechanisms for multi-strange baryons, and also for the baryon junction scheme discussed above. In PYTHIA, multistrange baryons are produced by string breakings, while in EPOS-LHC they mainly originate from

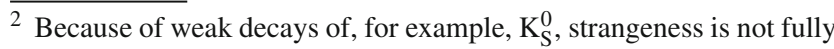
conserved in the final state.
} 

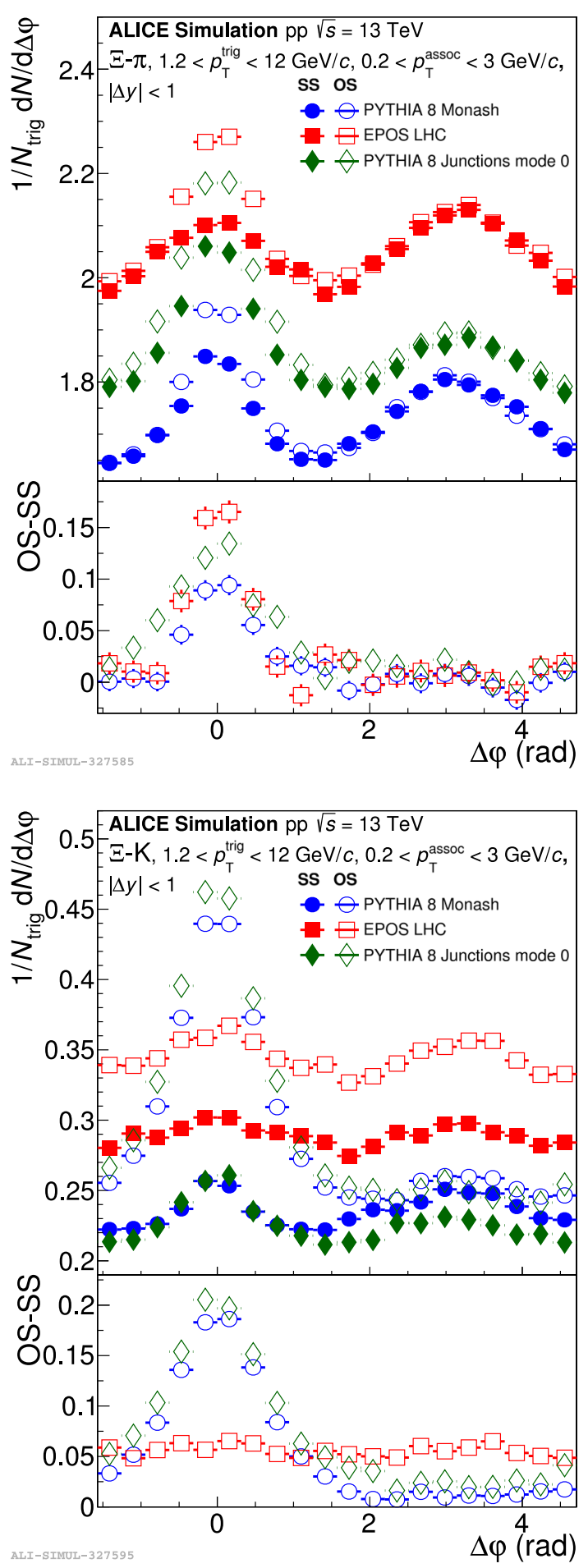

Fig. 10 Simulated $\Xi-\pi$ correlation (top) and $\Xi-K$ correlations (bottom). The upper panels show the correlations for opposite-(OS) and same-sign (SS) quantum numbers (charge top and strangeness bottom). The lower panel shows the difference (OS-SS), which is expected to measure the correlated production. The model calculations are PYTHIA8 in blue [17,93], EPOS-LHC in red [112], and a special PYTHIA8 baryon junction tune in green [113]. Copyright CERN, reused with permission

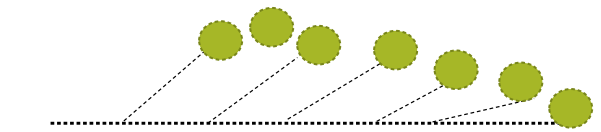

Shower in vacuum ...

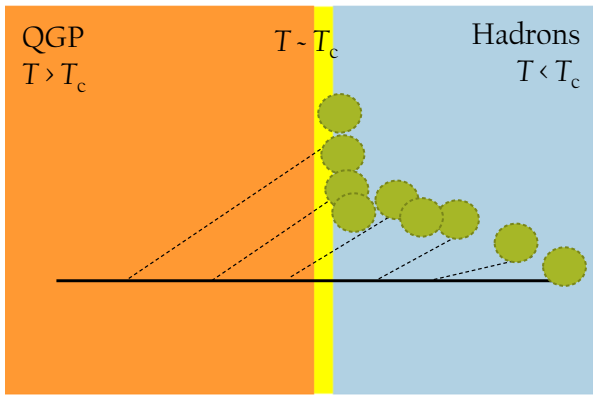

...and medium

Fig. 11 Cartoon of a parton shower creating a jet in vacuum (top) and in a deconfined medium (bottom). Green dots represent partons that hadronize. In the medium, partons associated with the jet shower have to propagate to the $T=T_{c}$ hypersurface before hadronizing

a QGP-like core. If one focuses on the bottom panels, one observes that all three calculations have different predictions for the pion and kaon yields associated with the production of a $\Xi$ baryon. The simulation results demonstrate the potential of future measurements and also highlights the care with which these correlations will have to be modeled.

\subsection{Spacetime structure of jets}

Jets present complementary opportunities to study QCD in general and hadronization in particular. High-momentum probes and jets have been studied over the past two decades as probes of QGP. New analyses of $e^{+} e^{-}$data [116], e.g., using modern jet substructure techniques, can also be used to constrain hadronization models.

One novel challenge of modeling jets is the role of the space-time structure of a jet and its relation to the spacetime structure of the underlying medium in nuclear collisions. Modern analysis techniques like re-clustering of jets and Lund plane analyses can provide glimpses of the splitting history of jet showers. Theoretical tools can further map these onto a space-time picture. Hadronization in A-A collisions is modified, as typically only part of the jet hadronizes similar to vacuum jets, while the remaining part is immersed in the QGP, see Fig. 11. This is a challenge to hadronization models. Strings attached to shower partons might reconnect or terminate with thermal partons. Recombination models have been developed to model hadronization with both shower and thermal partons as input $[117,118]$.

Space-time pictures need to be implemented into improved shower Monte Carlo and hadronization models in the future. New precise jet substructure analyses in $\mathrm{pp}, \mathrm{p}-\mathrm{A}$, and $\mathrm{A}-$ 
A, together with a reanalysis of $e^{+} e^{-}$data, will be able to provide important new constraints on hadronization models. Studies of high-momentum hadrons and jets in small systems as a function of multiplicity or event activity can be particularly illuminating.

\subsection{Summary}

Flavor correlations between hadrons seem to provide unique fingerprints to discriminate between string and rope models, on the one hand, and thermal models and recombination, on the other. We add two additional remarks.

- ALICE results suggest that strangeness production shows a very strong dependence on multiplicity [4]. Therefore it is critical to vary the multiplicity to test if the strangeness production mechanism changes as the strangeness is enhanced (or suppression is lifted).

- Correlation measurements of $\phi, \Xi$ and $\Omega$ hadrons are extremely statistics hungry and therefore Run 3 and 4 at LHC are perfect opportunities for ALICE to study them.

The statistical thermal model does not a priori give predictions for these correlations (although the notion of a correlation volume in the canonical ensemble can be introduced [119]). The non-locality of quantum number conservation seems a natural requirement for a deconfined and thermalized QGP medium. We encourage further theoretical work into the microscopic underpinning of these models.

In the jet sector, substructure observables, as well as the dependence of observables on system size and event multiplicity, will lead to challenges to existing hadronization models. Proper modeling of space-time properties will become increasingly important.

\section{Can heavy quarks unravel common mechanisms in small and large systems?}

The heavy charm and bottom quarks $(Q=\mathrm{c}, \mathrm{b})$ play a special role in the investigation of QCD dynamics. On the one hand, their masses are much larger than the typical QCD scale, $M_{Q} \gg \Lambda_{\mathrm{QCD}}$; on the other hand, their lifetimes are long enough to form hadronic bound states (although this does not hold for the top quark). This renders them excellent probes of: (a) hadronic structure in both open and hidden heavyflavor (HF) sectors (where the large mass facilitates approximation schemes such as non-relativistic QCD or potential approaches); (b) particle production mechanisms in elementary collision systems (e.g., testing heavy-quark (HQ) production and hadronization mechanisms); (c) nuclear effects in $\mathrm{p}-\mathrm{A}$ collisions (e.g., shadowing or quarkonium-absorption effects); (d) transport properties and hadronization of the deconfined medium in heavy-ion collisions [120].

Several puzzling observations in the HF sector have been made in pp, $\mathrm{p}-\mathrm{A}$, and $\mathrm{A}-\mathrm{A}$ collisions by experiments at RHIC and the LHC in recent years that call for investigations of seemingly related (or maybe unrelated) mechanisms. In pp collisions, a surprisingly large production yield of charm baryons has been reported [121], with a rather significant dependence on rapidity [122]. An enhancement of the $\Lambda_{\mathrm{c}} / D^{0}$ ratio has been measured in A-A collisions at RHIC [123], while it is less pronounced at the LHC [124]. For quarkonium production, an interesting dependence on multiplicity has been measured in pp collisions [125]; on the other hand, the enhancement in A-A collisions was expected by predictions of transport [126] and statistical hadronization [127] models, as a consequence of (re) combination of abundant anti-/charm quarks in the QGP and/or at the hadronization transition. In A-A collisions, the $p_{\mathrm{T}}$ spectra $[128,129]$ and azimuthal asymmetries of HF particles ( $D$ mesons and semileptonic decay leptons, and also charmonia) [130-134] have shown remarkable evidence for collectivity via the patterns in their nuclear modification factor $\left(R_{\mathrm{AA}}\right)$ and their large elliptic flow $\left(v_{2}\right)$, see upper panel in Fig. 12. For $D$ mesons, the $v_{2}$ is not far from that of light-flavor hadrons, providing direct evidence for a strong coupling of charm quarks to the expanding QGP medium [120]. However, a considerable $v_{2}$ for these particles has been observed as well in high-multiplicity $\mathrm{p}-\mathrm{Pb}$ collisions $[135,136]$ (see lower panel of Fig. 12), even though the QGP fireball, if any, is much smaller and shorter lived; furthermore, the pertinent $R_{\mathrm{AA}}$ shows only little modifications beyond cold-nuclear-matter (CNM) effects [137], except for the $\psi(2 S)$ [138]. In the following, we will report on recent discussions and insights on these issues, specifically for the kinetics (Sect. 6.1) and hadro-chemistry (Sect. 6.2) of openHF particles, followed by quarkonia (Sect. 6.3).

\subsection{The HF p-A puzzle}

The $D$-meson $v_{2}$ and $R_{\mathrm{AA}}$ in $5 \mathrm{TeV}$ p- $\mathrm{Pb}$ collisions have been investigated in Langevin simulations for "Brownian motion" of charm quarks and their hadronization assuming the presence of a hydrodynamically expanding medium $[139,140]$, with typical initial temperatures of near $400 \mathrm{MeV}$. While the predicted $v_{2}\left(p_{\mathrm{T}}\right)$ can reach values close to those observed in experiment [135] (see lower panel in Fig. 12) for a sufficiently strong $c$-quark-medium coupling, the calculated $R_{\mathrm{AA}}$ exhibits a low- $p_{\mathrm{T}}$ peak structure (as a consequence of the collective motion) and high- $p_{\mathrm{T}}$ suppression that does not agree with the essentially flat dependence of the data (which in turn is consistent with CNM effects only) [137]. One issue could be the validity of the Langevin approximation, if only one or two rescatterings occur in a p-A fireball. Even more extreme, it has been pointed out that in the limit of no rescat- 

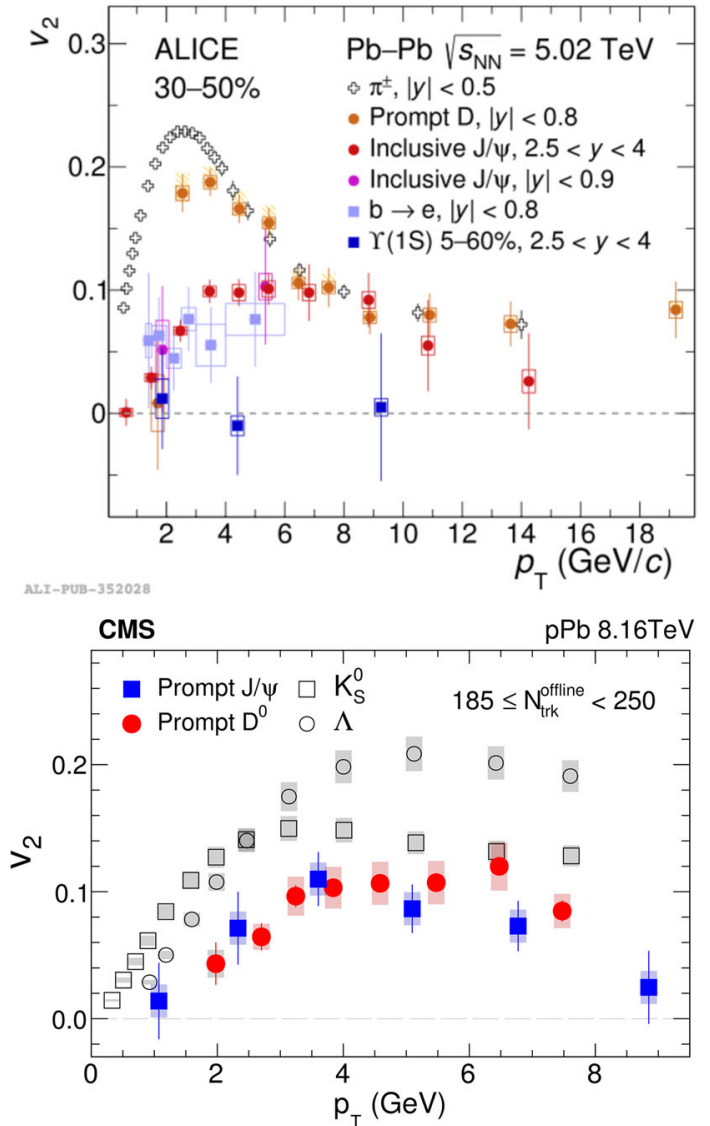

Fig. 12 Elliptic flow of open- and hidden-charm hadrons in $\mathrm{Pb}-\mathrm{Pb}$ collisions (upper panel, courtesy of the ALICE collaboration) and in high-multiplicity $\mathrm{p}-\mathrm{Pb}$ collisions at the LHC (lower panel, adopted from CMS [136])

tering, an escape effect along the short axis of the elliptic fireball can generate a positive $v_{2}$, although the quantitative effect is small for heavy quarks [145]. Nevertheless, revisiting HF transport in small systems using a kinetic (rather than hydrodynamic) bulk medium seems to be warranted. It is also of interest to aim for an improved measurement of the $D$-meson $v_{2}$ at low $p_{\mathrm{T}}$, in both $\mathrm{p}-\mathrm{A}$ and A-A collisions, which, in particular, can probe the presence of a negative dip as a tell-tale signature of a strong collective flow of heavy particles.

A recent calculation of the azimuthal asymmetry in heavyquarkonium production in $\mathrm{p}-\mathrm{Pb}$ collisions from initial-state effects [142] - specifically, the scattering of projectile partons off domains of differently oriented saturated gluon fields in the target nucleus - has found an elliptic flow consistent with ALICE $J / \psi$ data. The $v_{2}\left(p_{\mathrm{T}}\right)$ is predicted to be essentially identical for $J / \psi$ and $\Upsilon$. On the other hand, the ATLAS collaboration [143] has recently measured the $v_{2}$ of semileptonic decay dimuons in $13 \mathrm{TeV}$ pp collisions, separated into charm and bottom contributions and for $4<p_{\mathrm{T}}<6 \mathrm{GeV} / c$, finding a positive signal for charm but values consistent with zero for bottom.
We also recall that in semi-central $\mathrm{Pb}-\mathrm{Pb}$ collisions, the $J / \psi$ acquires a rather large $v_{2}$ of about 0.1 [134], whereas first data for $\Upsilon(1 S) v_{2}$ are compatible with zero [142,144], see upper panel in Fig. 12). These results are consistent with quarkonium transport models which predict a large $J / \psi$ regeneration component at low and intermediate $p_{\mathrm{T}}$ [126], while $\Upsilon(1 S)$ suppression - with a much smaller regeneration component - happens much earlier in the fireball evolution where the momentum anisotropy of the fireball is still small [146-148]. For $p_{\mathrm{T}} \gtrsim 6 \mathrm{GeV} / c$, however, the $J / \psi v_{2}$ data tend to be underestimated by the transport models, possibly due to space-momentum correlations of fast moving anti-/charm quarks [149] which have not been included in pertinent calculations yet.

\subsection{HF hadrochemistry}

The (soft) color neutralization of (hard-produced) heavy quarks provides an excellent window on hadronization mechanisms via the chemical composition of the produced HF hadrons. For non-strange $D$-mesons ( $D$ and $D^{*}$ ), the hadrochemistry does not show significant variations from pp to $\mathrm{p}-\mathrm{Pb}$ and $\mathrm{Pb}-\mathrm{Pb}$ collisions, and essentially follows that of a statistical hadronization with relative weights given by thermal factors at a "hadronization temperature" of $T_{H} \simeq$ $160 \mathrm{MeV}$. The $D_{\mathrm{s}} / D$ ratio is also compatible with this pattern, once a strangeness suppression factor of $\gamma_{\mathrm{s}} \simeq 0.6$, as independently inferred from strange-particle production, is accounted for in pp collisions [150]. In semi-/central A-A collisions $\gamma_{\mathrm{s}}$ is close to 1 (which is believed to be a consequence of strangeness equilibration in the QGP fireball), and the expected increase of the $D_{\mathrm{s}} / D$ ratio in A-A collisions [151-153] is indeed compatible with experimental observations [154]. The three-quark nature of baryons renders their spectrum of possible quantum numbers substantially richer, which in the hadron spectroscopy context gave rise to the problem of missing states in the measured spectra. Even rather recently, it has been argued [155] that QCD thermodynamics as computed in lattice QCD (1QCD) requires more strange-baryon states than currently listed by the particle data group (PDG) [156].

The knowledge of excited baryon states is much scarcer in the charm (and bottom) sector. Recent measurements by ALICE in 5 and $7 \mathrm{TeV}$ pp collisions at midrapidity have found a much larger $\Lambda_{\mathrm{c}} / D^{0}$ ratio of $\sim 0.54 \pm 0.16$ than previously measured, e.g., in $e^{+} e^{-}$annihilation $(\sim 0.1)$. It is also significantly larger than expectations from string-based event generators [115], as well as statistical hadronization ( 0.22) [151] when utilizing the known charm-baryon states as listed by the PDG. In Ref. [150] it was shown that upon including a largely augmented charm-baryon spectrum as predicted by relativistic quark models (also consistent with 1QCD [157]), the ALICE measurement could 
be accounted for. Improvements in the string fragmentation scenario, by accounting for color correlations beyond the leading-color approximation to create several MPI subsystems, can also facilitate the formation of charm baryons in pp collisions [113], yielding a $\Lambda_{c} / D^{0}$ ratio close to that measured at midrapidity. A potential caveat in both descriptions is that the LHCb measurement at forward rapidity finds a significantly smaller result, of about $0.25 \pm 0.05$ in pp [122] and $0.35 \pm 0.05$ [158] in $\mathrm{p}-\mathrm{Pb}$ collisions. These results could point at a multiplicity dependence of this ratio, saturating at the value given by the statistical hadronization model. A more microscopic understanding of how the multiplicity affects this ratio is clearly in order. Of high interest are measurements of additional charm baryons, which would also be very helpful to quantify the feed-down contributions to ground states. For example, recent data for $\Xi_{c}^{0}$ production in $7 \mathrm{TeV}$ pp collisions suggest a large enhancement relative to predictions of baseline event generators [159].

Let us finally comment on the current situation for charm-baryon production in A-A collisions. Recent STAR data [123] suggest a further increase of the $\Lambda_{\mathrm{c}} / D^{0}$ ratio in central $0.2 \mathrm{TeV} \mathrm{Au}-\mathrm{Au}$ collisions to $\sim 1.05 \pm 0.25$, compared to $\sim 0.5 \pm 0.1$ in peripheral collisions. On the other hand, in $\mathrm{Pb}-\mathrm{Pb}$ collisions at the $\mathrm{LHC}$, the centrality dependence is much less pronounced, with a rather small increase (if any) from pp to central $\mathrm{Pb}-\mathrm{Pb}$ collisions [124]. It remains to be seen whether there is a tension between these data, as theoretical predictions generally do not expect large differences between RHIC and the LHC $[149,160,161]$. The main uncertainty in these measurements is their reach to low momenta, which is currently limited to $p_{\mathrm{T}} \gtrsim 2 \mathrm{GeV} / c$. An accurate inclusive-yield measurement will be pivotal for addressing medium modifications of this ratio, e.g., to scrutinize the relation between the production mechanisms in pp and $\mathrm{p}-$ $\mathrm{Pb}$ collisions and to better understand the redistribution of the charm-hadron yields in $p_{\mathrm{T}}$ at hadronization; after all, the charm-quark spectra at the hadronization transition share the same modification due to their prior diffusion through the collectively expanding fireball medium. For example, spacemomentum correlations between fast-moving heavy quarks and thermal partons in the outer parts of the expanding fireball have recently been found to have a significant impact on both $R_{\mathrm{AA}}$ and $v_{2}$ observables of charm hadrons [149].

\subsection{Quarkonia}

The discovery of quarkonia in the 1970's established the basic QCD force in vacuum, with a perturbative color-Coulomb part at small distances and a linear "confining" potential taking over for distances of $r \gtrsim 0.2 \mathrm{fm}$. The linear potential dominates the binding for all charmonia and bottomonia, except the $\Upsilon(1 S)$ ground state. Consequently, their production systematics in A-A collisions plays a key role in deduc- ing the screening of the confining force in the medium and, more generally, its role in the properties of the QGP and its $\mathrm{de} /$ confinement transition. However, the originally envisaged suppression signature has significantly evolved over the last $\sim 15$ years, and it became clear that (re)generation processes in the hot QCD medium play a decisive role. The observed enhancement of $J / \psi$ production in $\mathrm{Pb}-\mathrm{Pb}$ collisions at the $\mathrm{LHC}$, relative to the large suppression in $\mathrm{Au}-\mathrm{Au}$ collisions at RHIC, was predicted by both transport models and the statistical hadronization model and corroborated by its concentration at low $p_{\mathrm{T}}$, as well as a large elliptic flow [126]. Significant uncertainties in the regeneration component remain due to current uncertainties in the total charm production cross section in $\mathrm{Pb}-\mathrm{Pb}$ collisions. To resolve those, low- $p_{\mathrm{T}}$ measurements of both $D$-meson and $\Lambda_{\mathrm{c}}$ production are essential, as also mentioned in the previous subsection. The key quarkonium transport parameter is the inelastic reaction rate, $\Gamma_{\mathcal{Q}}$, which is responsible for both their suppression and regeneration. In a recent work [162], it has been shown that bottomonia are a more promising observable than charmonia to infer the in-medium QCD force from their suppression pattern, due to a smaller "contamination" from regeneration. A statistical analysis of all available $\Upsilon R_{\mathrm{AA}}$ data from A-A collisions at RHIC and the LHC deduced a "strong" in-medium potential, with remnants of the confining force surviving well above $T_{\mathrm{c}}$. Interestingly, the same potential yields a heavy-quark diffusion coefficient which is in the right range to account for open-HF phenomenology [163]. Indeed, the quantitative coupling of open- and hidden-HF transport remains a challenging problem $[164,165]$. However, it has been argued that the $\Upsilon(2 S) / \Upsilon(1 S)$ ratio for central $\mathrm{Pb}-\mathrm{Pb}$ collisions may also be compatible with the statistical hadronization model [127]. Detailed measurements of weakly bound states, like $\psi(2 S)$, $\Upsilon(3 S)$, and even $X(3872)[166,167]$, including their $p_{\mathrm{T}}$ dependence and $v_{2}$, are promising observables to better disentangle the production mechanism.

A full understanding of the production mechanism of quarkonia in $\mathrm{pp}$ and $\mathrm{p}-\mathrm{Pb}$ collisions remains elusive thus far. This includes the multiplicity dependence of $J / \psi$ production, which increases substantially with $N_{\mathrm{ch}}$, stronger than linear (see Ref. [125] and references therein). The suppression of $J / \psi$ in $\mathrm{p}-\mathrm{Pb}$ is largely described in shadowing/saturation models, but the larger suppression of $\psi(2 S)$ [138], which is more prominent for higher-multiplicity events [168], remains not well understood to date, as is the case also for the observed $v_{2}$ of $J / \psi$ in high-multiplicity events [136], see lower panel in Fig. 12.

Acknowledgements The organizers would like to thank all the participants for making a both inspiring and enjoyable workshop, where many interesting new ideas were discussed and developed, some of which have been summarized in this document. The workshop was organized using funding for the project "CLASH: Pinning down the origin of collective effects in small collision systems". We want to thank the Knut and Alice 
Wallenberg Foundation for funding this project. This work was further supported by the Swedish VR contract No. 2017-003 (C. Bierlich), NCN under Grant No. 2018/29/B/ST2/00244 (P. Bozek), EU H2020 MSCA ITN Grant agreement MCnetITN3/722104 (S. Chakraborty and M. Utheim), FAPESP under Grant 17/05685-2 (D. D. Chinellato), US NSF awards PHY-1516590 and PHY-1812431 (R.J. Fries), PAPIIT-UNAM under Project No. IN102118 (A. Ortiz), US-DOE under award No. DE-SC0018117 (D.V. Perepelitsa), US-NSF under Grant no. PHY-1913286 (R. Rapp), and ERC H2020 Advanced Grant agreement No. 668679 (C. O. Rasmussen and T. Sjöstrand).

Funding Open access funding provided by Lund University.

Data Availability Statement This manuscript has no associated data or the data will not be deposited. [Authors' comment: As this is an overview paper, it does not contain new data.]

Open Access This article is licensed under a Creative Commons Attribution 4.0 International License, which permits use, sharing, adaptation, distribution and reproduction in any medium or format, as long as you give appropriate credit to the original author(s) and the source, provide a link to the Creative Commons licence, and indicate if changes were made. The images or other third party material in this article are included in the article's Creative Commons licence, unless indicated otherwise in a credit line to the material. If material is not included in the article's Creative Commons licence and your intended use is not permitted by statutory regulation or exceeds the permitted use, you will need to obtain permission directly from the copyright holder. To view a copy of this licence, visit http://creativecomm ons.org/licenses/by/4.0/.

\section{References}

1. J.L. Nagle, W.A. Zajc, Small system collectivity in relativistic hadronic and nuclear collisions. Ann. Rev. Nucl. Part. Sci. 68, (2018). https://doi.org/10.1146/annurev-nucl-101916-123209. arXiv:1801.03477 [nucl-ex]

2. CMS Collaboration, S. Chatrchyan et al., Observation and studies of jet quenching in $\mathrm{PbPb}$ collisions at nucleon-nucleon center-ofmass energy $=2.76 \mathrm{TeV}$. Phys. Rev. C 84, (2011). https://doi.org/ 10.1103/PhysRevC.84.024906. arXiv:1102.1957 [nucl-ex]

3. CMS Collaboration, S. Chatrchyan et al., Suppression of nonprompt $J / \psi$, prompt $J / \psi$, and $\mathrm{Y}(1 \mathrm{~S})$ in $\mathrm{PbPb}$ collisions at $\sqrt{s_{N N}}=2.76$ TeV. JHEP 05, (2012). https://doi.org/10.1007/ JHEP05(2012)063. arXiv:1201.5069 [nucl-ex]

4. ALICE Collaboration, J. Adam et al., Enhanced production of multi-strange hadrons in high-multiplicity proton-proton collisions. Nat. Phys. 13, (2017). https://doi.org/10.1038/nphys4111. arXiv:1606.07424 [nucl-ex]

5. ALICE Collaboration, K. Aamodt et al., Higher harmonic anisotropic flow measurements of charged particles in $\mathrm{Pb}-\mathrm{Pb}$ collisions at $\sqrt{s_{N N}}=2.76 \mathrm{TeV}$. Phys. Rev. Lett. 107, (2011). https:// doi.org/10.1103/PhysRevLett.107.032301. arXiv:1105.3865 [nucl-ex]

6. K. Dusling, F. Gelis, T. Lappi, R. Venugopalan, Long range twoparticle rapidity correlations in A+A collisions from high energy QCD evolution. Nucl. Phys. A 836, (2010). https://doi.org/10. 1016/j.nuclphysa.2009.12.044. arXiv:0911.2720 [hep-ph]

7. ALICE Collaboration, B. Abelev et al., Long-range angular correlations on the near and away side in $p-\mathrm{Pb}$ collisions at $\sqrt{s_{N N}}=$ 5.02 TeV. Phys. Lett. B 719, (2013). https://doi.org/10.1016/j. physletb.2013.01.012. arXiv:1212.2001 [nucl-ex]

8. CMS Collaboration, S. Chatrchyan et al., Observation of longrange near-side angular correlations in proton-lead collisions at the LHC. Phys. Lett. B 718, (2013). https://doi.org/10.1016/j. physletb.2012.11.025. arXiv:1210.5482 [nucl-ex]

9. ALICE Collaboration, S. Acharya et al., Multiplicity dependence of (multi-)strange hadron production in proton-proton collisions at $\sqrt{s}=13$ TeV. arXiv:1908.01861 [nucl-ex]

10. F. Gelis, E. Iancu, J. Jalilian-Marian, R. Venugopalan, The color Glass condensate. Ann. Rev. Nucl. Part. Sci. 60, (2010). https:// doi.org/10.1146/annurev.nucl.010909.083629. arXiv:1002.0333 [hep-ph]

11. A. Kurkela, A. Mazeliauskas, J.-F. Paquet, S. Schlichting, and D. Teaney, Matching the nonequilibrium initial stage of heavy ion collisions to hydrodynamics with QCD kinetic theory. Phys. Rev. Lett. 122 no. 12, (2019). https://doi.org/10.1103/PhysRevLett. 122.122302. arXiv: 1805.01604 [hep-ph]

12. A. Buckley et al., General-purpose event generators for LHC physics. Phys. Rep. 504, (2011). https://doi.org/10.1016/j. physrep.2011.03.005. arXiv:1101.2599 [hep-ph]

13. S. Jeon and U. Heinz, Introduction to hydrodynamics. Int. J. Mod. Phys. E 24(10), (2015). https://doi.org/10.1142/ S0218301315300106. arXiv:1503.03931 [hep-ph]

14. C. Shen, Z. Qiu, H. Song, J. Bernhard, S. Bass, and U. Heinz, The iEBE-VISHNU code package for relativistic heavy-ion collisions. Comput. Phys. Commun. 199, (2016). https://doi.org/10.1016/j. cpc.2015.08.039. arXiv:1409.8164 [nucl-th]

15. B. Schenke, S. Jeon, C. Gale, (3+1)D Hydrodynamic simulation of relativistic heavy-ion collisions. Phys. Rev. C 82, (2010). https://doi.org/10.1103/PhysRevC.82.014903. arXiv:1004.1408 [hep-ph]

16. C. Bierlich, G. Gustafson, L. Lönnblad, Collectivity without plasma in hadronic collisions. Phys. Lett. B 779, (2018). https:// doi.org/10.1016/j.physletb.2018.01.069. arXiv:1710.09725 [hep$\mathrm{ph}]$

17. T. Sjöstrand, S. Mrenna, and P.Z. Skands, PYTHIA 6.4 physics and manual. JHEP 05, (2006). https://doi.org/10.1088/ 1126-6708/2006/05/026. arXiv:hep-ph/0603175 [hep-ph]

18. C. Bierlich, G. Gustafson, L. Lönnblad, H. Shah, The Angantyr model for heavy-ion collisions in PYTHIA8. JHEP 10, (2018). https://doi.org/10.1007/JHEP10(2018)134. arXiv: 1806.10820 [hep-ph]

19. X.-N. Wang, M. Gyulassy, HIJING: a Monte Carlo model for multiple jet production in p p, p A and A A collisions. Phys. Rev. D 44, (1991). https://doi.org/10.1103/PhysRevD.44.3501

20. A.H. Mueller, Soft gluons in the infinite momentum wave function and the BFKL pomeron. Nucl. Phys. B 415, (1994). https://doi. org/10.1016/0550-3213(94)90116-3

21. C. Bierlich, C.O. Rasmussen, Dipole evolution: perspectives for collectivity and $\gamma^{*}$ A collisions. JHEP 10, (2019). https://doi.org/ 10.1007/JHEP10(2019)026. arXiv:1907.12871 [hep-ph]

22. B. Schenke, P. Tribedy, and R. Venugopalan, Fluctuating Glasma initial conditions and flow in heavy ion collisions. Phys. Rev. Lett. 108, (2012). https://doi.org/10.1103/PhysRevLett.108.252301. arXiv:1202.6646 [nucl-th]

23. ALICE Collaboration, S. Acharya et al., Centrality and pseudorapidity dependence of the charged-particle multiplicity density in $\mathrm{Xe}-\mathrm{Xe}$ collisions at $\sqrt{s_{\mathrm{NN}}}=5.44 \mathrm{TeV}$. Phys. Lett. B 790, (2019). https://doi.org/10.1016/j.physletb.2018.12.048. arXiv:1805.04432 [nucl-ex]

24. CMS Collaboration, A.M. Sirunyan et al., Pseudorapidity distributions of charged hadrons in xenon-xenon collisions at $\sqrt{s_{\mathrm{NN}}}=$ 5.44 TeV. Phys. Lett. B 799, (2019). https://doi.org/10.1016/j. physletb.2019.135049. arXiv:1902.03603 [hep-ex]

25. PHOBOS Collaboration, B. Alver et al., Phobos results on charged particle multiplicity and pseudorapidity distributions in $\mathrm{Au}+\mathrm{Au}$, $\mathrm{Cu}+\mathrm{Cu}, \mathrm{d}+\mathrm{Au}$, and $\mathrm{p}+\mathrm{p}$ collisions at ultra-relativistic energies. Phys. Rev. C 83, (2011). https://doi.org/10.1103/PhysRevC.83. 024913. arXiv:1011.1940 [nucl-ex] 
26. ATLAS Collaboration, M. Aaboud et al., Measurement of longrange multiparticle azimuthal correlations with the subevent cumulant method in $p p$ and $p+P b$ collisions with the ATLAS detector at the CERN large hadron collider. Phys. Rev. C 97 no. 2, (2018). https://doi.org/10.1103/PhysRevC.97.024904. arXiv: 1708.03559 [hep-ex]

27. CMS Collaboration, V. Khachatryan et al., Evidence for collectivity in $\mathrm{pp}$ collisions at the LHC. Phys. Lett. B 765, (2017). https://doi.org/10.1016/j.physletb.2016.12.009. arXiv:1606.06198 [nucl-ex]

28. W. Zhao, Y. Zhou, H. Xu, W. Deng, and H. Song, Hydrodynamic collectivity in proton-proton collisions at $13 \mathrm{TeV}$. Phys. Lett. B 780, (2018). https://doi.org/10.1016/j.physletb.2018.03. 022. arXiv:1801.00271 [nucl-th]

29. R.S. Bhalerao and J.-Y. Ollitrault, Eccentricity fluctuations and elliptic flow at RHIC. Phys. Lett. B 641, (2006). https://doi.org/10. 1016/j.physletb.2006.08.055. arXiv:nucl-th/0607009 [nucl-th]

30. W. Zhao, Y. Zhou, K. Murase, and H. Song, Searching for small droplets of hydrodynamic fluid in proton-proton collisions at the LHC. arXiv:2001.06742 [nucl-th]

31. B. Blok, C.D. Jökel, M. Strikman, U.A. Wiedemann, Collectivity from interference. JHEP 12, (2017). https://doi.org/10.1007/ JHEP12(2017)074. arXiv:1708.08241 [hep-ph]

32. CMS Collaboration, A.M. Sirunyan et al., Multiparticle correlation studies in $\mathrm{pPb}$ collisions at $\sqrt{s_{\mathrm{NN}}}=8.16 \mathrm{TeV}$. Phys. Rev. C 101 no. 1, (2020). https://doi.org/10.1103/PhysRevC.101. 014912. arXiv:1904.11519 [hep-ex]

33. ALICE Collaboration, S. Acharya et al., Investigations of anisotropic flow using multiparticle azimuthal correlations in $\mathrm{pp}, \mathrm{p}-\mathrm{Pb}, \mathrm{Xe}-\mathrm{Xe}$, and $\mathrm{Pb}-\mathrm{Pb}$ collisions at the LHC. Phys. Rev. Lett. 123 no. 14, (2019). https://doi.org/10.1103/PhysRevLett. 123.142301. arXiv:1903.01790 [nucl-ex]

34. ALICE Collaboration, J. Adam et al., Correlated event-by-event fluctuations of flow harmonics in $\mathrm{Pb}-\mathrm{Pb}$ collisions at $\sqrt{s_{\mathrm{NN}}}=$ 2.76 TeV. Phys. Rev. Lett. 117, (2016). https://doi.org/10.1103/ PhysRevLett.117.182301. arXiv:1604.07663 [nucl-ex]

35. CMS Collaboration, A.M. Sirunyan et al., Observation of correlated azimuthal anisotropy Fourier harmonics in $p p$ and $p+P b$ collisions at the LHC. Phys. Rev. Lett. 120 no. 9, (2018). https://doi.org/10.1103/PhysRevLett.120.092301. arXiv:1709.09189 [nucl-ex]

36. C. Mordasini, A. Bilandzic, D. Karakoç, and S.F. Taghavi, Higher order symmetric cumulants. Phys. Rev. C 102, (2020). https://doi. org/10.1103/PhysRevC.102.024907. arXiv:1901.06968 [nuclex]

37. C. Bierlich et al., Robust independent validation of experiment and theory: rivet version 3. arXiv:1912.05451 [hep-ph]

38. M.A. Lisa, S. Pratt, R. Soltz, and U. Wiedemann, Femtoscopy in relativistic heavy ion collisions. Ann. Rev. Nucl. Part. Sci. 55, (2005). https://doi.org/10.1146/annurev.nucl.55.090704.151533. arXiv:nucl-ex/0505014 [nucl-ex]

39. A.N. Makhlin, Y.M. Sinyukov, Hydrodynamics of hadron matter under pion interferometric microscope. Z. Phys. C 39, (1988). https://doi.org/10.1007/BF01560393. ([238 (1987)])

40. T. Csorgo and B. Lorstad, Bose-Einstein correlations for expanding finite systems. Nucl. Phys. A 590, (1995). https://doi.org/10. 1016/0375-9474(95)00255-Y. arXiv:hep-ph/9503494 [hep-ph]

41. T. Csorgo and B. Lorstad, Bose-Einstein correlations for threedimensionally expanding, cylindrically symmetric, finite systems. Phys. Rev. C 54, (1996). https://doi.org/10.1103/PhysRevC.54. 1390. arXiv:hep-ph/9509213 [hep-ph]

42. U.W. Heinz, B. Tomasik, U.A. Wiedemann, and Y.F. Wu, Kaon interferometry. Acta Phys. Hung. A 4, (1996). arXiv:nucl-th/9606041 [nucl-th]

43. ALICE Collaboration, K. Aamodt et al., Femtoscopy of $p p$ collisions at $\sqrt{s}=0.9$ and $7 \mathrm{TeV}$ at the LHC with two-pion Bose-
Einstein correlations. Phys. Rev. D 84, (2011). https://doi.org/10. 1103/PhysRevD.84.112004. arXiv:1101.3665 [hep-ex]

44. ALICE Collaboration, J. Adam et al., Two-pion femtoscopy in $\mathrm{p}-\mathrm{Pb}$ collisions at $\sqrt{s_{\mathrm{NN}}}=5.02 \mathrm{TeV}$. Phys. Rev. C 91, (2015). https://doi.org/10.1103/PhysRevC.91.034906. arXiv:1502.00559 [nucl-ex]

45. B. Schenke and R. Venugopalan, Eccentric protons? Sensitivity of flow to system size and shape in $\mathrm{p}+\mathrm{p}, \mathrm{p}+\mathrm{Pb}$ and $\mathrm{Pb}+\mathrm{Pb}$ collisions. Phys. Rev. Lett. 113, (2014). https://doi.org/10.1103/ PhysRevLett.113.102301. arXiv:1405.3605 [nucl-th]

46. ALICE Collaboration, B.B. Abelev et al., Multiplicity dependence of Pion, Kaon, proton and lambda production in $\mathrm{p}-\mathrm{Pb}$ collisions at $\sqrt{s_{N N}}=5.02 \mathrm{TeV}$. Phys. Lett. B 728, (2014). https://doi.org/ 10.1016/j.physletb.2013.11.020. arXiv:1307.6796 [nucl-ex]

47. ATLAS Collaboration, G. Aad et al., Observation of longrange elliptic azimuthal anisotropies in $\sqrt{s}=13$ and $2.76 \mathrm{TeV}$ $p p$ collisions with the ATLAS Detector. Phys. Rev. Lett. 116 no. 17, (2016). https://doi.org/10.1103/PhysRevLett.116.172301. arXiv: 1509.04776 [hep-ex]

48. G. Gustafson, How can we discriminate between a (cold) dense colour field or overlapping strings and a (hot) quarkgluon plasma. https://indico.lucas.lu.se/event/1214/contribution/ 36/material/poster/0.pdf

49. C. Bierlich, G. Gustafson, L. Lönnblad, A. Tarasov, Effects of overlapping strings in pp collisions. JHEP 03, (2015). https://doi. org/10.1007/JHEP03(2015)148. arXiv:1412.6259 [hep-ph]

50. A. Bilandzic, C.H. Christensen, K. Gulbrandsen, A. Hansen, and Y. Zhou, Generic framework for anisotropic flow analyses with multiparticle azimuthal correlations. Phys. Rev. C 89(6), (2014). https://doi.org/10.1103/PhysRevC.89.064904. arXiv:1312.3572 [nucl-ex]

51. ATLAS Collaboration, M. Aaboud et al., Correlated long-range mixed-harmonic fluctuations measured in $p p, p+\mathrm{Pb}$ and lowmultiplicity $\mathrm{Pb}+\mathrm{Pb}$ collisions with the ATLAS detector. Phys. Lett. B 789, (2019). https://doi.org/10.1016/j.physletb.2018.11. 065. arXiv:1807.02012 [nucl-ex]

52. J. Adolfsson, Study of strangeness enhancement in small systems through $\Xi$-hadron correlations in pp collisions at $13 \mathrm{TeV}$. https://indico.lucas.lu.se/event/1214/contribution/35/ material/poster/0.pdf

53. L. He, T. Edmonds, Z.-W. Lin, F. Liu, D. Molnar, and F. Wang, Anisotropic parton escape is the dominant source of azimuthal anisotropy in transport models. Phys. Lett. B 753, (2016). https://doi.org/10.1016/j.physletb.2015.12.051. arXiv:1502.05572 [nucl-th]

54. A. Mazeliauskas and V. Vislavicius, Temperature and fluid velocity on the freeze-out surface from $\pi, K, p$ spectra in $\mathrm{pp}, \mathrm{p}-\mathrm{Pb}$ and $\mathrm{Pb}-\mathrm{Pb}$ collisions. Phys. Rev. C 101(1), (2020). https://doi.org/10. 1103/PhysRevC.101.014910. arXiv:1907.11059 [hep-ph]

55. W. Zhao, C.M. Ko, Y.-X. Liu, G.-Y. Qin, and H. Song, Probing the partonic degrees of freedom in high multiplicity $\mathrm{p}-\mathrm{Pb}$ collisions at $\sqrt{s_{N N}}=5.02 \mathrm{TeV}$. arXiv: 1911.00826 [nucl-th]

56. R. Katz, C.A.G. Prado, J. Noronha-Hostler, and A.A.P. Suaide, System size scan of $\mathrm{D}$ meson $R_{\mathrm{AA}}$ and $v_{n}$ using $\mathrm{PbPb}, \mathrm{XeXe}$, ArAr, and OO collisions at LHC. arXiv:1907.03308 [nucl-th]

57. A. Ohlson, Short-range correlations in small systems. https:// indico.lucas.lu.se/event/1214/contribution/25/material/poster/0. pdf

58. A. Ortiz, What does pythia tell us about jets surrounded by extremely high underlying event?. https://indico.lucas.lu.se/ event/1214/contribution/37/material/poster/0.pdf

59. D.J. Kim, Ridge in hard events in pp collisions and jet parton shower in high multiplicity ppb collisions. https://indico.lucas.lu. se/event/1214/contribution/44/material/poster/0.pdf

60. L. Frankfurt, M. Strikman, and C. Weiss, Dijet production as a centrality trigger for $p p$ collisions at CERN LHC. Phys. 
Rev. D 69, (2004). https://doi.org/10.1103/PhysRevD.69.114010. arXiv:hep-ph/0311231 [hep-ph]

61. A. Kurkela and Y. Zhu, Isotropization and hydrodynamization in weakly coupled heavy-ion collisions. Phys. Rev. Lett. 115(18), (2015). https://doi.org/10.1103/PhysRevLett.115. 182301. arXiv:1506.06647 [hep-ph]

62. Z.-W. Lin, Evolution of transverse flow and effective temperatures in the parton phase from a multi-phase transport model. Phys. Rev. C 90(1), (2014). https://doi.org/10.1103/PhysRevC. 90.014904. arXiv:1403.6321 [nucl-th]

63. ALICE Collaboration, J. Adam et al., Higher harmonic flow coefficients of identified hadrons in $\mathrm{Pb}-\mathrm{Pb}$ collisions at $\sqrt{s_{\mathrm{NN}}}=2.76$ TeV. JHEP 09, (2016). https://doi.org/10.1007/ JHEP09(2016)164. arXiv:1606.06057 [nucl-ex]

64. N. Borghini and C. Gombeaud, Anisotropic flow far from equilibrium. Eur. Phys. J. C 71, (2011). https://doi.org/10.1140/epjc/ s10052-011-1612-7. arXiv:1012.0899 [nucl-th]

65. N. Borghini, S. Feld, and N. Kersting, Scaling behavior of anisotropic flow harmonics in the far from equilibrium regime. Eur. Phys. J. C 78 no. 10, (2018). https://doi.org/10.1140/epjc/ s10052-018-6313-z. arXiv:1804.05729 [nucl-th]

66. A. Kurkela, U.A. Wiedemann, and B. Wu, Opacity dependence of elliptic flow in kinetic theory. Eur. Phys. J. C 79(9), (2019). https://doi.org/10.1140/epjc/s10052-019-7262-x. arXiv:1805.04081 [hep-ph]

67. Y. Sun, S. Plumari, and V. Greco, Study of collective anisotropies $v_{2}$ and $v_{3}$ and their fluctuations in $p A$ collisions at LHC within a relativistic transport approach. Eur. Phys. J. C 80(1), (2020). https://doi.org/10.1140/epjc/s10052-019-7577-7. arXiv:1907.11287 [nucl-th]

68. K. Tywoniuk, Is there jet quenching in $\mathrm{pPb}$ ? Nucl. Phys. A 926 , (2014). https://doi.org/10.1016/j.nuclphysa.2014.04.023

69. A. Kurkela, U.A. Wiedemann, and B. Wu, Flow in AA and $\mathrm{pA}$ as an interplay of fluid-like and non-fluid like excitations. Eur. Phys. J. C 79(11), (2019). https://doi.org/10.1140/epjc/ s10052-019-7428-6. arXiv:1905.05139 [hep-ph]

70. H.A. Andrews et al., Novel tools and observables for jet physics in heavy-ion collisions. arXiv:1808.03689 [hep-ph]

71. CMS Collaboration, A.M. Sirunyan et al., Measurement of the splitting function in $p p$ and $\mathrm{Pb}-\mathrm{Pb}$ collisions at $\sqrt{s_{\mathrm{NN}}}=5.02$ TeV. Phys. Rev. Lett. 120(14), (2018). https://doi.org/10.1103/ PhysRevLett.120.142302. arXiv:1708.09429 [nucl-ex]

72. ALICE Collaboration, S. Acharya et al., Exploration of jet substructure using iterative declustering in $\mathrm{pp}$ and $\mathrm{Pb}-\mathrm{Pb}$ collisions at LHC energies. arXiv:1905.02512 [nucl-ex]

73. D.A. Appel, Jets as a probe of quark-gluon plasmas. Phys. Rev. D 33, (1986)

74. J.P. Blaizot, L.D. McLerran, Jets in expanding quark-gluon plasmas. Phys. Rev. D 34, (1986)

75. ALICE Collaboration, J. Adam et al., Measurement of jet quenching with semi-inclusive hadron-jet distributions in central $\mathrm{Pb}-\mathrm{Pb}$ collisions at $\sqrt{s_{\mathrm{NN}}}=2.76 \mathrm{TeV}$. JHEP 09 (2015). arXiv:1506.03984 [nucl-ex]

76. STAR Collaboration, L. Adamczyk et al., "Measurements of jet quenching with semi-inclusive hadron+jet distributions in $\mathrm{Au}+\mathrm{Au}$ collisions at $\sqrt{s_{N N}}=200 \mathrm{GeV}$ ", Phys. Rev. C 96 no. 2, (2017). arXiv:1702.01108 [nucl-ex]

77. L. Chen, G.-Y. Qin, S.-Y. Wei, B.-W. Xiao, H.-Z. Zhang, Probing transverse momentum broadening via dihadron and hadron-jet angular correlations in relativistic heavy-ion collisions. Phys. Lett. B 773, (2017). arXiv:1607.01932 [hep-ph]

78. ALICE Collaboration, J. Adam et al., Centrality dependence of particle production in $\mathrm{p}-\mathrm{Pb}$ collisions at $\sqrt{s_{\mathrm{NN}}}=5.02 \mathrm{TeV}$. Phys. Rev. C 91(6), (2015). https://doi.org/10.1103/PhysRevC. 91.064905. arXiv:1412.6828 [nucl-ex]
79. M.L. Miller, K. Reygers, S.J. Sanders, and P. Steinberg, Glauber modeling in high energy nuclear collisions. Ann. Rev. Nucl. Part. Sci. 57, (2007). https://doi.org/10.1146/annurev.nucl.57.090506. 123020. arXiv:nucl-ex/0701025 [nucl-ex]

80. D.V. Perepelitsa and P.A. Steinberg, Calculation of centrality bias factors in $p+\mathrm{A}$ collisions based on a positive correlation of hard process yields with underlying event activity. arXiv:1412.0976 [nucl-ex]

81. A. Ortiz and L. Valencia Palomo, Probing color reconnection with underlying event observables at the LHC energies. Phys. Rev. D 99(3), (2019). https://doi.org/10.1103/PhysRevD.99.034027. arXiv:1809.01744 [hep-ex]

82. PHENIX Collaboration, A. Adare et al., Centrality categorization for $R_{p(d)+A}$ in high-energy collisions. Phys. Rev. C 90(3), (2014). https://doi.org/10.1103/PhysRevC.90.034902. arXiv:1310.4793 [nucl-ex]

83. PHENIX Collaboration, A. Adare et al., Centrality-dependent modification of jet-production rates in deuteron-gold collisions at $\sqrt{s_{N N}}=200 \mathrm{GeV}$. Phys. Rev. Lett. 116(12), (2016). https://doi. org/10.1103/PhysRevLett.116.122301. arXiv:1509.04657 [nuclex]

84. ATLAS Collaboration, G. Aad et al., Centrality and rapidity dependence of inclusive jet production in $\sqrt{s_{\mathrm{NN}}}=5.02$ $\mathrm{TeV}$ proton-lead collisions with the ATLAS detector. Phys. Lett. B 748 (2015). https://doi.org/10.1016/j.physletb.2015.07. 023. arXiv:1412.4092 [hep-ex]

85. ALICE Collaboration, J. Adam et al., Centrality dependence of charged jet production in $\mathrm{p}-\mathrm{Pb}$ collisions at $\sqrt{s_{\mathrm{NN}}}=5.02$ TeV. Eur. Phys. J. C 76(5), (2016). https://doi.org/10.1140/epjc/ s10052-016-4107-8. arXiv:1603.03402 [nucl-ex]

86. ALICE Collaboration, S. Acharya et al., Transverse momentum spectra and nuclear modification factors of charged particles in pp, p-Pb and Pb-Pb collisions at the LHC. JHEP 11 (2018). https:// doi.org/10.1007/JHEP11(2018)013. arXiv:1802.09145 [nucl-ex]

87. ATLAS Collaboration, G. Aad et al., Transverse momentum, rapidity, and centrality dependence of inclusive charged-particle production in $\sqrt{s_{N N}}=5.02 \mathrm{TeV} p+\mathrm{Pb}$ collisions measured by the ATLAS experiment. Phys. Lett. B 763 (2016). https://doi.org/ 10.1016/j.physletb.2016.10.053. arXiv:1605.06436 [hep-ex]

88. CMS Collaboration, V. Khachatryan et al., Charged-particle nuclear modification factors in $\mathrm{PbPb}$ and $\mathrm{pPb}$ collisions at $\sqrt{s_{\mathrm{NN}}}=5.02 \mathrm{TeV}$. JHEP 04 (2017). https://doi.org/10.1007/ JHEP04(2017)039. arXiv:1611.01664 [nucl-ex]

89. ALICE Collaboration, S. Acharya et al., Constraints on jet quenching in $\mathrm{p}-\mathrm{Pb}$ collisions at $\sqrt{\mathbf{S}_{\mathbf{N N}}}=5.02 \mathrm{TeV}$ measured by the event-activity dependence of semi-inclusive hadron-jet distributions. Phys. Lett. B 783 (2018). https://doi.org/10.1016/j. physletb.2018.05.059. arXiv:1712.05603 [nucl-ex]

90. B. Schenke, C. Gale, S. Jeon, MARTINI: Monte Carlo simulation of jet evolution. Acta Phys. Polon. Supp. 3, (2010). arXiv:0911.4470 [hep-ph]

91. K.C. Zapp, JEWEL 2.0.0: directions for use. Eur. Phys. J. C 74(2), (2014). https://doi.org/10.1140/epjc/s10052-014-2762-1. arXiv:1311.0048 [hep-ph]

92. M. Bahr et al., Herwig++ Physics and Manual. Eur. Phys. J. C 58, (2008). https://doi.org/10.1140/epjc/s10052-008-0798-9. arXiv:0803.0883 [hep-ph]

93. T. Sjöstrand, S. Ask, J.R. Christiansen, R. Corke, N. Desai, P. Ilten, S. Mrenna, S. Prestel, C.O. Rasmussen, P.Z. Skands, An introduction to PYTHIA 8.2. Comput. Phys. Commun. 191, (2015). https://doi.org/10.1016/j.cpc.2015.01.024. arXiv: 1410.3012 [hep-ph]

94. T. Gleisberg, S. Hoeche, F. Krauss, M. Schonherr, S. Schumann, F. Siegert, and J. Winter, Event generation with SHERPA 1.1. JHEP 02 (2009). https://doi.org/10.1088/1126-6708/2009/ 02/007. arXiv:0811.4622 [hep-ph] 
95. J. Bellm, C.B. Duncan, S. Gieseke, M. Myska, and A. Siódmok, Spacetime colour reconnection in Herwig 7. Eur. Phys. J. C 79(12), (2019). https://doi.org/10.1140/epjc/ s10052-019-7533-6. arXiv:1909.08850 [hep-ph]

96. T. Sjöstrand, M. van Zijl, A multiple interaction model for the event structure in hadron collisions. Phys. Rev. D 36, (1987)

97. ALICE Collaboration, P.M. Jacobs, Search for jet quenching effects in high multiplicity pp collisions at $\sqrt{\mathrm{s}}=13 \mathrm{TeV}$, in 28th International Conference on Ultrarelativistic Nucleus-Nucleus Collisions (Quark Matter 2019) Wuhan, China, November 4-9, 2019. (2020). arXiv:2001.09517 [nucl-ex]

98. G. Paić, Pythia and data revisited at very high multiplicity and number of events in the free Interpretation of Guy Paic. https://indico.lucas.lu.se/event/1214/contribution/30/ material/poster/0.pdf

99. A.N. Mishra and G. Paić, Parton energy loss in pp collisions at very high multiplicity. arXiv:1905.06918 [hep-ph]

100. H. Heiselberg, G. Baym, B. Blaettel, L.L. Frankfurt, M. Strikman, Color transparency, color opacity, and fluctuations in nuclear collisions. Phys. Rev. Lett. 67, (1991). https://doi.org/10.1103/ PhysRevLett.67.2946

101. B. Blaettel, G. Baym, L.L. Frankfurt, H. Heiselberg, M. Strikman, Hadronic cross-section fluctuations. Phys. Rev. D 47, (1993). https://doi.org/10.1103/PhysRevD.47.2761

102. M. Alvioli, M. Strikman, Color fluctuation effects in protonnucleus collisions. Phys. Lett. B 722, (2013). https://doi.org/10. 1016/j.physletb.2013.04.042. arXiv:1301.0728 [hep-ph]

103. M. Alvioli, L. Frankfurt, V. Guzey, M. Strikman, Revealing "flickering" of the interaction strength in pA collisions at the CERN LHC. Phys. Rev. C 90, (2014). https://doi.org/10.1103/ PhysRevC.90.034914. arXiv:1402.2868 [hep-ph]

104. M. Alvioli, B.A. Cole, L. Frankfurt, D.V. Perepelitsa, and M. Strikman, Evidence for $x$-dependent proton color fluctuations in pA collisions at the CERN Large Hadron Collider. Phys. Rev. C 93(1), (2016). https://doi.org/10.1103/PhysRevC.93.011902. arXiv:1409.7381 [hep-ph]

105. E. Avsar, G. Gustafson, and L. Lönnblad, Energy conservation and saturation in small-x evolution. JHEP 07, (2005). https://doi.org/ 10.1088/1126-6708/2005/07/062. arXiv:hep-ph/0503181 [hep$\mathrm{ph}]$

106. E. Avsar, G. Gustafson, and L. Lönnblad, Small-x dipole evolution beyond the large-N(c) imit. JHEP 01, (2007). https://doi.org/10. 1088/1126-6708/2007/01/012. arXiv:hep-ph/0610157 [hep-ph]

107. C. Flensburg, G. Gustafson, L. Lönnblad, Inclusive and exclusive observables from dipoles in high energy collisions. JHEP 08, (2011). https://doi.org/10.1007/JHEP08(2011)103. arXiv:1103.4321 [hep-ph]

108. B. Andersson, G. Gustafson, B. Nilsson-Almqvist, A model for low $\mathrm{p}(\mathrm{t})$ hadronic reactions, with generalizations to hadronnucleus and nucleus-nucleus collisions. Nucl. Phys. B 281, (1987). https://doi.org/10.1016/0550-3213(87)90257-4

109. B. Andersson, G. Gustafson, G. Ingelman, T. Sjöstrand, Parton fragmentation and string dynamics. Phys. Rep. 97, (1983). https:// doi.org/10.1016/0370-1573(83)90080-7

110. F. Becattini, R. Fries, The QCD confinement transition: hadron formation. Landolt-Bornstein 23, (2010). https://doi.org/10.1007/ 978-3-642-01539-7_8. arXiv:0907.1031 [nucl-th]

111. R608 Collaboration, A.M. Smith et al., Evidence for pomeron single quark interactions in proton diffraction at the ISR. Phys. Lett. B 163, (1985). https://doi.org/10.1016/0370-2693(85)90235-7

112. T. Pierog, I. Karpenko, J.M. Katzy, E. Yatsenko, and K. Werner, EPOS LHC: Test of collective hadronization with data measured at the CERN large hadron collider. Phys. Rev. C 92(3), (2015). https://doi.org/10.1103/PhysRevC.92.034906. arXiv:1306.0121 [hep-ph]
113. J.R. Christiansen, P.Z. Skands, String formation beyond leading colour. JHEP 08, (2015). https://doi.org/10.1007/ JHEP08(2015)003. arXiv:1505.01681 [hep-ph]

114. ALICE Collaboration, J. Adam et al., Insight into particle production mechanisms via angular correlations of identified particles in pp collisions at $\sqrt{\mathrm{s}}=7 \mathrm{TeV}$. Eur. Phys. J. C 77(8), (2017). https://doi.org/10.1140/epjc/s10052-017-5129-6. arXiv:1612.08975 [nucl-ex]. [Erratum: Eur.Phys.J.C 79, 998 (2019)]

115. C. Bierlich and J.R. Christiansen, Effects of color reconnection on hadron flavor observables. Phys. Rev. D 92(9), (2015). https://doi. org/10.1103/PhysRevD.92.094010. arXiv:1507.02091 [hep-ph]

116. A. Badea, A. Baty, P. Chang, G.M. Innocenti, M. Maggi, C. Mcginn, M. Peters, T.-A. Sheng, J. Thaler, and Y.-J. Lee, Measurements of two-particle correlations in $e^{+} e^{-}$collisions at $91 \mathrm{GeV}$ with ALEPH archived data. Phys. Rev. Lett. 123(21), (2019). https://doi.org/10.1103/PhysRevLett.123. 212002. arXiv:1906.00489 [hep-ex]

117. K.C. Han, R.J. Fries, and C.M. Ko, Jet fragmentation via recombination of parton showers. Phys. Rev. C 93(4), (2016). https://doi. org/10.1103/PhysRevC.93.045207. arXiv:1601.00708 [nucl-th]

118. R.J. Fries and M. Kordell, Hybrid hadronization. PoS HardProbes2018 (2019). https://doi.org/10.22323/1.345.0046. arXiv:1901.08157 [nucl-th]

119. S. Hamieh, K. Redlich, and A. Tounsi, Canonical description of strangeness enhancement from $\mathrm{p}-\mathrm{A}$ to $\mathrm{Pb} \mathrm{Pb}$ collisions. Phys. Lett. B 486 (2000). https://doi.org/10.1016/S0370-2693(00)00762-0. arXiv:hep-ph/0006024 [hep-ph]

120. F. Prino and R. Rapp, Open heavy flavor in QCD matter and in nuclear collisions. J. Phys. G 43(9), (2016). https://doi.org/10. 1088/0954-3899/43/9/093002. arXiv:1603.00529 [nucl-ex]

121. ALICE Collaboration, S. Acharya et al., $\Lambda_{c}^{+}$production in pp collisions at $\sqrt{s}=7 \mathrm{TeV}$ and in $\mathrm{p}-\mathrm{Pb}$ collisions at $\sqrt{s_{\mathrm{NN}}}=5.02 \mathrm{TeV}$. JHEP 04, (2018). https://doi.org/10.1007/ JHEP04(2018)108. arXiv:1712.09581 [nucl-ex]

122. LHCb Collaboration, R. Aaij et al., "Prompt charm production in pp collisions at $\operatorname{sqrt}(\mathrm{s})=7 \mathrm{TeV}$ ", Nucl. Phys. B 871 (2013). https://doi.org/10.1016/j.nuclphysb.2013.02.010. arXiv:1302.2864 [hep-ex]

123. STAR Collaboration, J. Adam et al., First measurement of $\wedge c$ baryon production in in $\mathrm{Au}+\mathrm{Au}$ collisions at $\sqrt{s_{N N}}=200 \mathrm{GeV}$. Phys. Rev. Lett. 124, 172301 (2020)

124. ALICE Collaboration, C. Zampolli, "talk at strangeness in quark matter 2019." https://indico.cern.ch/event/755366/ sessions/311861/\#20190611

125. ALICE Collaboration, S. Acharya et al., Multiplicity dependence of $\mathrm{J} / \psi$ production at midrapidity in pp collisions at $\sqrt{s}=13 \mathrm{TeV}$. arXiv:2005.11123 [nucl-ex]

126. R. Rapp, X. Du, Theoretical perspective on quarkonia from SPS via RHIC to LHC. Nucl. Phys. A 967, (2017). https://doi.org/10. 1016/j.nuclphysa.2017.05.097. arXiv:1704.07923 [hep-ph]

127. A. Andronic, P. Braun-Munzinger, K. Redlich, and J. Stachel, Decoding the phase structure of QCD via particle production at high energy. Nature 561(7723), (2018). https://doi.org/10.1038/ s41586-018-0491-6. arXiv:1710.09425 [nucl-th]

128. CMS Collaboration, A.M. Sirunyan et al., Nuclear modification factor of $\mathrm{D}^{0}$ mesons in $\mathrm{PbPb}$ collisions at $\sqrt{s_{\mathrm{NN}}}=5.02 \mathrm{TeV}$. Phys. Lett. B 782, (2018). https://doi.org/10.1016/j.physletb.2018.05. 074. arXiv:1708.04962 [nucl-ex]

129. ALICE Collaboration, F. Grosa, Measurement of D-meson nuclear modification factor and flow in $\mathrm{Pb}-\mathrm{Pb}$ collisions with ALICE at the LHC. PoS HardProbes2018, (2018). https://doi. org/10.22323/1.345.0138. arXiv:1812.06188 [nucl-ex]

130. CMS Collaboration, A.M. Sirunyan et al., Measurement of prompt $D^{0}$ meson azimuthal anisotropy in $\mathrm{Pb}-\mathrm{Pb}$ collisions at $\sqrt{s_{N N}}=$ 
5.02 TeV. Phys. Rev. Lett. 120(20), (2018). https://doi.org/10. 1103/PhysRevLett.120.202301. arXiv:1708.03497 [nucl-ex]

131. ALICE Collaboration, S. Acharya et al., Elliptic flow of electrons from beauty-hadron decays in $\mathrm{Pb}-\mathrm{Pb}$ collisions at $\sqrt{s_{\mathrm{NN}}}=5.02$ TeV. arXiv:2005.11130 [nucl-ex]

132. ALICE Collaboration, S. Acharya et al., Transverse-momentum and event-shape dependence of $\mathrm{D}$-meson flow harmonics in $\mathrm{Pb}-$ $\mathrm{Pb}$ collisions at $\sqrt{s_{N N}}=5.02 \mathrm{TeV}$. arXiv:2005.11131 [nucl-ex]

133. CMS Collaboration, V. Khachatryan et al., Suppression and azimuthal anisotropy of prompt and nonprompt $\mathrm{J} / \psi$ production in $\mathrm{PbPb}$ collisions at $\sqrt{s_{\mathrm{NN}}}=2.76 \mathrm{TeV}$. Eur. Phys. J. C 77(4), (2017). https://doi.org/10.1140/epjc/s10052-017-4781-1. arXiv: 1610.00613 [nucl-ex]

134. ALICE Collaboration, S. Acharya et al., $\mathrm{J} / \psi$ elliptic and triangular flow in $\mathrm{Pb}-\mathrm{Pb}$ collisions at $\sqrt{s_{\mathrm{NN}}}=5.02 \mathrm{TeV}$. arXiv:2005.14518 [nucl-ex]

135. CMS Collaboration, A.M. Sirunyan et al., Elliptic flow of charm and strange hadrons in high-multiplicity $\mathrm{pPb}$ collisions at $\sqrt{s_{\mathrm{NN}}}=$ 8.16 TeV. Phys. Rev. Lett. 121(8), (2018). https://doi.org/10.1103/ PhysRevLett.121.082301. arXiv:1804.09767 [hep-ex]

136. CMS Collaboration, A.M. Sirunyan et al., Observation of prompt $\mathrm{J} / \psi$ meson elliptic flow in high-multiplicity $\mathrm{pPb}$ collisions at $\sqrt{s_{\mathrm{NN}}}=8.16 \mathrm{TeV}$. Phys. Lett. B 791, (2019). https://doi.org/ 10.1016/j.physletb.2019.02.018. arXiv:1810.01473 [hep-ex]

137. ALICE Collaboration, S. Acharya et al., Measurement of prompt $\mathrm{D}^{0}, \mathrm{D}^{+}, \mathrm{D}^{*+}$, and $\mathrm{D}_{\mathrm{S}}^{+}$production in $\mathrm{p}-\mathrm{Pb}$ collisions at $\sqrt{\mathrm{s}_{\mathrm{NN}}}=5.02 \mathrm{TeV}$. JHEP 12, (2019). https://doi.org/10.1007/ JHEP12(2019)092. arXiv:1906.03425 [nucl-ex]

138. ALICE Collaboration, B.B. Abelev et al., Suppression of $\psi(2 \mathrm{~S})$ production in $\mathrm{p}-\mathrm{Pb}$ collisions at $\sqrt{s_{\mathrm{NN}}}=5.02 \mathrm{TeV}$. JHEP 12, (2014). https://doi.org/10.1007/JHEP12(2014)073. arXiv:1405.3796 [nucl-ex]

139. A. Beraudo, A. De Pace, M. Monteno, M. Nardi, F. Prino, Heavyflavour production in high-energy $\mathrm{d}-\mathrm{Au}$ and $\mathrm{p}-\mathrm{Pb}$ collisions. JHEP 03, (2016). https://doi.org/10.1007/JHEP03(2016)123. arXiv: 1512.05186 [hep-ph]

140. Y. Xu, S. Cao, G.-Y. Qin, W. Ke, M. Nahrgang, J. Auvinen, and S.A. Bass, Heavy-flavor dynamics in relativistic p$\mathrm{Pb}$ collisions at $\sqrt{S_{N N}}=5.02 \mathrm{TeV}$. Nucl. Part. Phys. Proc. 276, (2016). https://doi.org/10.1016/j.nuclphysbps.2016.05.050. arXiv: 1510.07520 [nucl-th]

141. H. Li, Z.-W. Lin, F. Wang, Charm quarks are more hydrodynamic than light quarks in final-state elliptic flow. Phys. Rev. C 99(4), 044911 (2019). https://inspirehep.net/literature/1666852. arXiv:1804.02681 [hep-ph]

142. C. Zhang, C. Marquet, G.-Y. Qin, S.-Y. Wei, and B.-W. Xiao, Elliptic flow of heavy quarkonia in $p A$ collisions. Phys. Rev. Lett. 122(17), (2019). https://doi.org/10.1103/PhysRevLett.122. 172302. arXiv:1901.10320 [hep-ph]

143. ATLAS Collaboration, G. Aad et al., Measurement of azimuthal anisotropy of muons from charm and bottom hadrons in $p p$ collisions at $\sqrt{s}=13 \mathrm{TeV}$ with the ATLAS detector. Phys. Rev. Lett. 124, 082301 (2020). arXiv:1909.01650 [nucl-ex]

144. ALICE Collaboration, S. Acharya et al., Measurement of $\Upsilon$ (1S) elliptic flow at forward rapidity in $\mathrm{Pb}-\mathrm{Pb}$ collisions at $\sqrt{s_{\mathrm{NN}}}=$ 5.02 TeV. Phys. Rev. Lett. 123(19), (2019). https://doi.org/10. 1103/PhysRevLett.123.192301. arXiv:1907.03169 [nucl-ex]

145. CMS Collaboration, Measurement of the elliptic flow of $\Upsilon(1 \mathrm{~S})$ and $\Upsilon(2 \mathrm{~S})$ mesons in $\mathrm{PbPb}$ collisions at $\sqrt{\mathrm{s}_{\mathrm{NN}}}=5.02 \mathrm{TeV}$. In: Tech. Rep. CMS-PAS-HIN-19-002, CERN, Geneva (2019). http://cds.cern.ch/record/2698580

146. X. Du, R. Rapp, and M. He, Color screening and regeneration of Bottomonia in high-energy heavy-ion collisions. Phys. Rev. C 96(5), (2017). https://doi.org/10.1103/PhysRevC.96.054901. arXiv:1706.08670 [hep-ph]
147. P.P. Bhaduri, N. Borghini, A. Jaiswal, and M. Strickland, Anisotropic escape mechanism and elliptic flow of bottomonia. Phys. Rev. C 100(5), (2019). https://doi.org/10.1103/PhysRevC. 100.051901. arXiv:1809.06235 [hep-ph]

148. J. Hong and S.H. Lee, $\Upsilon(1 S)$ transverse momentum spectra through dissociation and regeneration in heavy-ion collisions. Phys. Lett. B 801, (2020). https://doi.org/10.1016/j.physletb. 2019.135147. arXiv:1909.07696 [nucl-th]

149. M. He and R. Rapp, Hadronization and charm-hadron ratios in heavy-ion collisions. Phys. Rev. Lett. 124(4), (2020). https://doi. org/10.1103/PhysRevLett.124.042301. arXiv:1905.09216 [nuclth]

150. M. He and R. Rapp, Charm-baryon production in proton-proton collisions. Phys. Lett. B 795, (2019). https://doi.org/10.1016/j. physletb.2019.06.004. arXiv:1902.08889 [nucl-th]

151. A. Andronic, P. Braun-Munzinger, K. Redlich, and J. Stachel, Statistical hadronization of charm in heavy ion collisions at SPS, RHIC and LHC. Phys. Lett. B 571, (2003). https://doi.org/10. 1016/j.physletb.2003.07.066. arXiv:nucl-th/0303036 [nucl-th]

152. I. Kuznetsova and J. Rafelski, Heavy flavor hadrons in statistical hadronization of strangeness-rich QGP. Eur. Phys. J. C 51, (2007). https://doi.org/10.1140/epjc/s10052-007-0268-9. arXiv:hep-ph/0607203 [hep-ph]

153. M. He, R.J. Fries, and R. Rapp, $\mathbf{D}_{\mathbf{s}}$-Meson as quantitative probe of diffusion and hadronization in nuclear collisions. Phys. Rev. Lett. 110(11), (2013). https://doi.org/10.1103/PhysRevLett.110. 112301. arXiv: 1204.4442 [nucl-th]

154. ALICE Collaboration, $\mathrm{S}$. Acharya et al., Measurement of $\mathrm{D}^{0}, \mathrm{D}^{+}$, $\mathrm{D}^{*+}$ and $\mathrm{D}_{s}^{+}$production in $\mathrm{Pb}-\mathrm{Pb}$ collisions at $\sqrt{\mathrm{S}_{\mathrm{NN}}}=5.02 \mathrm{TeV}$. JHEP 10, (2018). https://doi.org/10.1007/JHEP10(2018)174. arXiv:1804.09083 [nucl-ex]

155. P. Alba et al., Constraining the hadronic spectrum through QCD thermodynamics on the lattice. Phys. Rev. D 96(3), (2017). https:// doi.org/10.1103/PhysRevD.96.034517. arXiv:1702.01113 [heplat]

156. Particle Data Group Collaboration, M. Tanabashi et al., Review of Particle Physics. Phys. Rev. D 98(3), (2018). https://doi.org/ 10.1103/PhysRevD.98.030001

157. P. Madanagopalan, R.G. Edwards, N. Mathur, and M.J. Peardon, Spectroscopy of charmed baryons from lattice QCD. PoS LATTICE2014, (2015). https://doi.org/10.22323/1.214.0084. arXiv: 1410.8791 [hep-lat]

158. LHCb Collaboration, $\mathrm{R}$. Aaij et al., Prompt $\Lambda_{c}^{+}$production in $p \mathrm{~Pb}$ collisions at $\sqrt{s_{N N}}=5.02 \mathrm{TeV}$. JHEP 02, (2019). https://doi.org/ 10.1007/JHEP02(2019)102. arXiv:1809.01404 [hep-ex]

159. ALICE Collaboration, S. Acharya et al., First measurement of $\Xi_{\mathrm{c}}^{0}$ production in pp collisions at $\sqrt{\mathbf{s}}=7 \mathrm{TeV}$. Phys. Lett. B 781, (2018). https://doi.org/10.1016/j.physletb.2018.03.061. arXiv: 1712.04242 [hep-ex]

160. J. Zhao, S. Shi, N. Xu, P. Zhuang, Sequential coalescence with charm conservation in high energy nuclear collisions. arXiv: 1805.10858 [hep-ph]

161. S. Plumari, V. Minissale, S.K. Das, G. Coci, and V. Greco, Charmed hadrons from coalescence plus fragmentation in relativistic nucleus-nucleus collisions at RHIC and LHC. Eur. Phys. J. C 78(4), (2018). https://doi.org/10.1140/epjc/ s10052-018-5828-7. arXiv:1712.00730 [hep-ph]

162. X. Du, S.Y.F. Liu, and R. Rapp, Extraction of the heavy-quark potential from bottomonium observables in heavy-ion collisions. Phys. Lett. B 796, (2019). https://doi.org/10.1016/j.physletb. 2019.07.032. arXiv:1904.00113 [nucl-th]

163. S.Y.F. Liu, M. He, and R. Rapp, Probing the in-medium QCD force by open heavy-flavor observables. Phys. Rev. C 99(5), (2019). https://doi.org/10.1103/PhysRevC.99.055201. arXiv:1806.05669 [nucl-th] 
164. P.B. Gossiaux, R. Katz, Upsilon suppression in the SchrödingerLangevin approach. Nucl. Phys. A 956, (2016). https://doi.org/ 10.1016/j.nuclphysa.2016.04.017. arXiv:1601.01443 [hep-ph]

165. N. Krenz, H. van Hees, and C. Greiner, Quarkonia production in a Langevin approach. J. Phys. Conf. Ser. 1070(1), (2018). https:// doi.org/10.1088/1742-6596/1070/1/012008. arXiv:1805.10798 [nucl-th]

166. ExHIC Collaboration, S. Cho et al., Exotic hadrons from heavy ion collisions. Prog. Part. Nucl. Phys. 95, (2017). https://doi.org/ 10.1016/j.ppnp.2017.02.002. arXiv:1702.00486 [nucl-th]
167. CMS Collaboration, Evidence for $\chi_{c 1}(3872)$ in $\mathrm{PbPb}$ collisions and studies of its prompt production at $\sqrt{s_{\mathrm{NN}}}=5.02 \mathrm{TeV}$, in Tech. Rep. CMS-PAS-HIN-19-005, CERN, Geneva, 2019. http:// cds.cern.ch/record/2698963

168. ALICE Collaboration, J. Adam et al., Centrality dependence of $\psi(2 \mathrm{~S})$ suppression in $\mathrm{p}-\mathrm{Pb}$ collisions at $\sqrt{s_{\mathrm{NN}}}=5.02 \mathrm{TeV}$. JHEP 06, (2016). https://doi.org/10.1007/JHEP06(2016)050. arXiv:1603.02816 [nucl-ex] 